DOI: $10.24850 /$ j-tyca-2020-06-07

Artículos

\title{
Generación de índices de seguridad hídrica municipal con un enfoque probabilista
}

\section{Generation of municipal water security indices by a probabilistic approach}

Felipe Ignacio Arreguín-Cortés ${ }^{1}$, ORCID: https://orcid.org/0000-00026577-6497

José Manuel Rodríguez-Varela2, ORCID: https://orcid.org/0000-0002$5509-2876$

Velitchko G. Tzatchkov3, ORCID: https://orcid.org/0000-0003-20818179

Petronilo Edilburgo Cortez-Mejía4, ORCID: https://orcid.org/0000-00019173-7515

Óscar Jesús Llaguno-Guilberto5, ORCID: https://orcid.org/0000-00029239-8081

Edgar Yuri Mendoza-Cázares6, ORCID: https://orcid.org/0000-00032127-6068

Arizabeth Sainos-Candelario7, ORCID: https://orcid.org/0000-0002-14582676

Juan Fco. Gómez-Martínez8, ORCID: https://orcid.org/0000-0002-65776497 
David Ortega-Gaucín9, ORCID: https://orcid.org/0000-0002-5336-7442 José Raúl Saavedra-Horita10

${ }^{1}$ Consultor, México, farreguin2011@gmail.com

2Instituto Mexicano de Tecnología del Agua, Jiutepec, Morelos, México, manuel_rodriguez@tlaloc.imta.mx

${ }^{3}$ Instituto Mexicano de Tecnología del Agua, Jiutepec, Morelos, México, velitchk@tlaloc.imta.mx

${ }^{4}$ Instituto Mexicano de Tecnología del Agua, Jiutepec, Morelos, México, pcortes@tlaloc.imta.mx

5Instituto Mexicano de Tecnología del Agua, Jiutepec, Morelos, México, oscar_llaguno@tlaloc.imta.mx

${ }^{6}$ Instituto Mexicano de Tecnología del Agua, Jiutepec, Morelos, México, edgar_mendoza@tlaloc.imta.mx

7Instituto Mexicano de Tecnología del Agua, Jiutepec, Morelos, México, arizabeth_sainos@tlaloc.imta.mx

8Instituto Mexicano de Tecnología del Agua, Jiutepec, Morelos, México, juanfco@tlaloc.imta.mx

9Instituto Mexicano de Tecnología del Agua, Jiutepec, Morelos, México, dortega@tlaloc.imta.mx

${ }^{10}$ Consultor, México, horita_2005@yahoo.com.mx

Autor para correspondencia: Velitchko Gueorguiev-Tzatchkov, velitchk@tlaloc.imta.mx 
Tecnología y

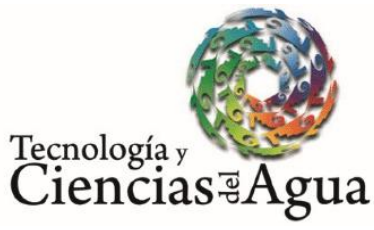

2020, Instituto Mexicano de Tecnología del Agua

Open Access bajo la licencia CC BY-NC-SA 4.0

(https://creativecommons.org/licenses/by-nc-sa/4.0/)

\section{Resumen}

En el siglo XXI, el agua escasea en Latinoamérica y el Caribe por el aumento de la demanda, resultado de un crecimiento poblacional, incremento de la producción de alimentos y mayor consumo energético, vinculados con una variación temporal del clima. Los gobiernos deben establecer programas de seguridad hídrica, a través de la planeación de la gestión del recurso agua, como un cambio del paradigma. México no es la excepción, un país de fuertes contrastes meteorológicos, hidrográficos y sociales a lo largo de su territorio, que afectan de varias formas a los diferentes estratos de la población, similar a otros países en desarrollo. En este contexto, se proponen y cuantifican índices de peligro, vulnerabilidad social y riesgo mediante una metodología probabilística para el cálculo del índice de seguridad hídrica. El peligro se expresa por indicadores como acceso a los servicios básicos de agua, inundaciones, abatimiento del nivel freático y sequias. A su vez, la vulnerabilidad contempla proporción de médicos por cada 1000 habitantes, tasa de mortalidad infantil, población derechohabiente, analfabetismo, condiciones de la vivienda, población económicamente activa, razón de dependencia, tasa de desempleo abierto y población de habla indígena. En el desarrollo de este estudio se demuestra que tanto el peligro como la vulnerabilidad a lo largo del territorio mexicano son muy heterogéneos. La metodología probabilística propuesta se apoyó en un sistema de información geográfica, lo que permitió obtener índices de seguridad hídrica para todos los municipios de México (2 456 municipios). 
Palabras clave: seguridad hídrica a nivel municipal, peligro, vulnerabilidad, riesgo.

\begin{abstract}
Water is turning to be scarce in Latin America and the Caribbean, in the 21st century, caused by an increase in its demand due to population growth, increased food production and higher energy consumption, linked to a temporary climate variation. Governments must establish water security programs by planning the management of water resources, as a paradigm shift. Mexico is not the exception, a country of strong meteorological, hydrographic and social contrasts throughout its territory, which impact in different ways the population strata, similar to other developing countries. In this context, hazard, social vulnerability and risk indexes are proposed and quantified in this paper, through a probabilistic methodology for calculating water security. Hazard is expressed by basic water services shortage, floods, groundwater depletion, and drought indicators. In turn, the vulnerability considers educational level of the population, access to health services, infant mortality rate, illiteracy, housing conditions, unemployment and proportion of indigenous language population. In the development of this study it is shown that both hazard and vulnerability throughout the Mexican territory are very heterogeneous. The proposed probabilistic methodology was implemented in a Geographical Information System obtaining water security indexes for all municipalities in Mexico (2,456 municipalities).
\end{abstract}

Keywords: Water security, municipalities, probability, indices, risk, vulnerability. 
Tecnología y

Ciencias $₫$ Agua

Recibido: 26/04/2019

Aceptado: 08/04/2020
2020, Instituto Mexicano de Tecnología del Agua

Open Access bajo la licencia CCBY-NC-SA 4.0

(https://creativecommons.org/licenses/by-nc-sa/4.0/)

\section{Introducción}

México ha tenido un crecimiento poblacional acelerado en los últimos años, que acarrea diferentes problemáticas, como proporcionar los diferentes tipos de servicios públicos: agua, gas, electricidad, entre otros, a los principales centros urbanos del país. Ante esto se necesita un manejo de los recursos naturales de manera sostenible, tratando de minimizar su impacto en el medio ambiente.

Actualmente existen diversos problemas en torno al agua: acuíferos sobreexplotados, contaminación y falta de saneamiento de cuerpos de agua, acceso desigual a los servicios básicos del agua y deterioro en la infraestructura hidráulica, entre otros. Por estas razones, la atención en la gestión de los recursos naturales y, en la particular, del agua, debe ser empleada de manera racional.

La vulnerabilidad social de las personas juega un papel importante en el manejo de los recursos, debido a que en el país los estratos sociales están muy marcados; por ejemplo, el Instituto Nacional de Estadística 
Geografía e Informática (INEGI, 2019) menciona que 43.6\% del total de la población de México está en condiciones de pobreza, e identifica a 26.8 millones de personas con carencias sociales.

La mayor frecuencia y severidad de los eventos climáticos extremos (sequías e inundaciones) genera la necesidad de realizar una gestión proactiva de los recursos hídricos para enfrentar dichas amenazas (GWP, 2012).

La creciente demanda y escasez del agua que se vive hoy en día requiere plantear un objetivo estratégico que contemple la gestión de los recursos hídricos, por lo que recientemente se ha incorporado el concepto de seguridad hídrica, que ha sido tema de numerosas reuniones y acuerdos internacionales (ONU-AGUA, 2013). Sin embargo, su definición difiere entre los distintos autores de acuerdo con los problemas que se pretenden solventar.

Lo anterior conlleva a que la definición de seguridad hídrica debe conceptualizar de una manera real la problemática que se vive, esto permitirá el desarrollo de una definición más precisa.

\section{Antecedentes}

Existen diferentes definiciones de seguridad hídrica. En México, MartínezAustria (2013) precisa el término como aquella condición que asegura el 
abastecimiento sustentable de agua para todos los usos en condiciones de equidad y a precios asequibles, para promover la salud, el desarrollo económico, la producción de alimentos, energía y la conservación del medio ambiente. Autores como Mason y Roger (2012) agregan que este término debe manejar los riesgos asociados con el agua, incluyendo inundaciones, sequías y contaminación; (Norman, Bakker, Cook, Dunn y Allen (2010) adicionan una protección en cantidad suficiente a los ecosistemas.

La Organización de las Naciones Unidas (ONU-AGUA, 2013), así como la Global Water Partnership (GWP, 2016) añaden a lo anterior que se debe asegurar la protección en contra de la contaminación transmitida por el agua y los desastres relacionados con ella, en un clima de paz y estabilidad política.

En 2017 (IMTA, 2017) y 2018 (IMTA, 2018), el Instituto Mexicano de Tecnología del Agua (IMTA) desarrolló el proyecto Índices de seguridad hídrica (ISH), en el que se definió, con base en información de diversas dependencias como el INEGI, el Centro Nacional de Prevención de Desastres (Cenapred) y la Comisión Nacional del Agua (Conagua), una metodología para determinar la seguridad hídrica por municipio en toda la república mexicana, con base en un análisis probabilístico de ocurrencia de algún fenómeno meteorológico, y a partir de la capacidad de la población para enfrentarlo; se representa mediante mapas de peligro, vulnerabilidad, riesgo y seguridad hídrica, como se indica a continuación. 
Tecnología y

Ciencias $₫$ Agua
2020, Instituto Mexicano de Tecnología del Agua

Open Access bajo la licencia CC BY-NC-SA 4.0

(https://creativecommons.org/licenses/by-nc-sa/4.0/)

\section{Materiales y métodos}

Entre las metodologías existentes para la medición del índice de seguridad hídrica se encuentra una aplicada a la región Asia-Pacífico (AWDO, 2013), que contabiliza el índice por medio de la satisfacción de los hogares (en saneamiento y agua potable); la capacidad de abastecimiento en la agricultura, minería, industria y energía, para obtener un beneficio productivo, además del apoyo de los servicios relacionados con el agua para la mejora de su calidad.

Mason y Roger (2012) proponen agrupar el índice en cinco rubros: 1) capacidad para tener acceso al recurso hídrico; 2) gestión de la variabilidad y riesgo; 3) satisfacción de las necesidades humanas; 4) atención de los requerimientos ambientales, y 5) gestión de la competencia y el conflicto relacionados con el aprovechamiento del recurso hídrico. Ait-Kadi y Linckaen (2016) plantean el indicador con base en tres dimensiones: sociales, ambientales y económicas

Gain, Giupponi y Wada (2016) propusieron un índice global de seguridad hídrica (IGSH) para el objetivo seis, de los 17 objetivos globales para Desarrollo Sostenible establecidos por la Organización de las Naciones Unidas (ONU, 2017).

En el presente trabajo se proponen índices de seguridad hídrica en el ámbito municipal, con un enfoque probabilista que tome en cuenta el peligro de fenómenos antrópicos y vulnerabilidad de la población. 


\section{Cálculo del índice de peligro}

El índice de peligro se cuantifica en términos de la probabilidad de que ocurra un fenómeno en un tiempo determinado, que sea potencialmente dañino para los bienes expuestos, o que se tengan problemas de acceso a los servicios de agua potable y saneamiento. El peligro se describe como las fuerzas, condiciones y tecnologías que conllevan un potencial daño social, infraestructural o ambiental, como un huracán, terremoto o avalancha (Oliver-Smith, \& Hoffman, 2002). Para este estudio, el peligro se integró por cuatro indicadores que se describen a continuación.

\section{Índice de falta de acceso a los servicios de agua y saneamiento $I_{\text {fasas }}$}

Para calcular el índice de peligro, los parámetros que se consideraron son acceso a los servicios de agua potable y saneamiento, definido, a su vez, mediante el índice global de acceso a los servicios básicos de agua (IGASA), que permite evaluar el impacto de la cobertura, calidad y 
eficiencia de los servicios de agua potable y saneamiento. Este índice es evaluado a partir de los siguientes indicadores: 1) acceso a los servicios de agua potable (IAAP), calculado con la información referente a cobertura urbana de agua potable (\%), cobertura rural de agua potable (\%) y agua desinfectada (\%); 2) acceso a los servicios de saneamiento (IAS), que se compone de la cobertura urbana de alcantarillado (\%), cobertura rural de alcantarillado (\%), eficiencia de recolección del agua residual generada (\%) y cobertura de tratamiento de aguas residuales municipales (\%).

Para integrar toda la información, las variables se normalizaron con respecto a sus valores mínimos y máximos de acuerdo con la Ecuación 1:

$Z_{i j}=\frac{X_{i j}-X_{\min }}{X_{\max }-X_{\min }}$

donde:

$Z_{i j}=$ variable normalizada.

$X_{i j}=$ variable asociada.

$X_{\min }=$ valor mínimo de los datos de la variable.

$X_{\max }=$ valor máximo de los datos de la variable.

$i=1$ a $n$.

$j=$ valor de la variable $i$ para la unidad de análisis.

$n=$ número de variables involucradas en el índice. 
Tecnología y

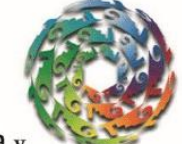

Ciencias ฐึgua
2020, Instituto Mexicano de Tecnología del Agua

Open Access bajo la licencia CC BY-NC-SA 4.0

(https://creativecommons.org/licenses/by-nc-sa/4.0/)

Por último, el índice de falta de acceso a los servicios de agua y saneamiento $I_{\text {fasas }}$ se expresa en la Ecuación 2. El resultado se muestra en la Figura 1.

$I_{\text {fasas }}=(1-I G A S A)$

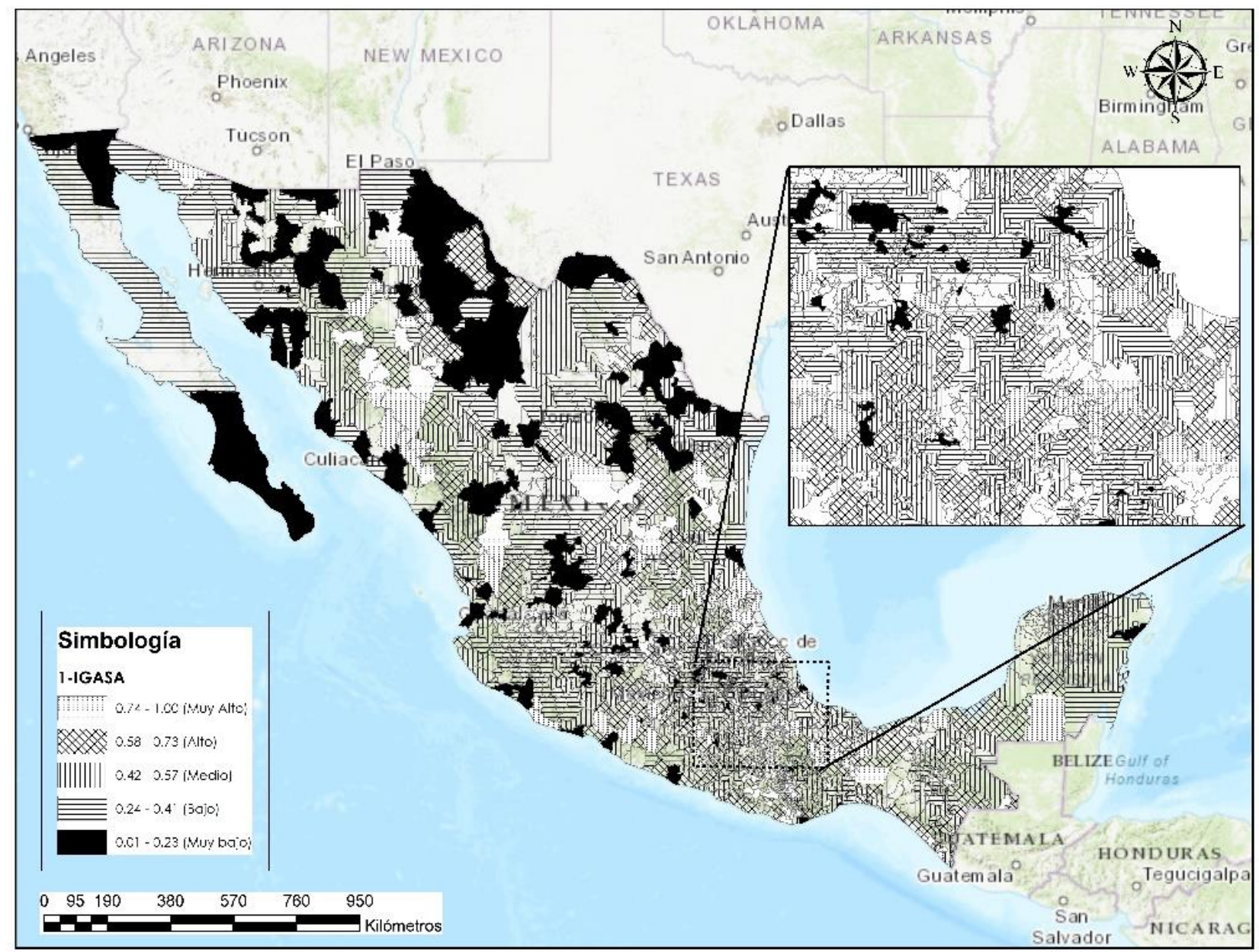

Figura 1. Mapa índice de falta de acceso a los servicios de agua y saneamiento por municipio $\boldsymbol{I}_{\text {fasas }}$. 
Ciencias đecnologí y
2020, Instituto Mexicano de Tecnología del Agua

Open Access bajo la licencia CC BY-NC-SA 4.0

(https://creativecommons.org/licenses/by-nc-sa/4.0/)

En la Figura 1 se identifican los municipios con mayores problemas de suministro y acceso a los servicios de agua potable y alcantarillado tanto de las zonas urbanas como rurales. Sobresale el estado de Oaxaca, con deficiencias en $83 \%$ de sus municipios, es decir, 476 municipios están en condiciones regulares, deficientes o muy deficientes. Por su extensión, Chihuahua tiene la mayor área en la zona "muy deficiente" de este indicador (1-IGASA), le siguen Oaxaca y Durango.

En la Tabla 1 se muestra el número de municipios que están en los diferentes rangos en que se dividió el indicador $I_{\text {fasas }}$. En resumen, se puede decir que $3.5 \%$ de la población tiene problemas de acceso a los servicios de agua potable, pues tales servicios y los de alcantarillado son muy deficientes; esta población se ubica en 250 municipios.

Tabla 1. Falta de acceso los servicios de agua potable y saneamiento $I_{\text {fasas }}$.

\begin{tabular}{|l|c|c|c|c|c|c|}
\hline \multicolumn{1}{|c|}{ Rangos } & $\begin{array}{c}\text { Número de } \\
\text { municipios }\end{array}$ & $\begin{array}{c}\text { Porcentaje } \\
\mathbf{d e} \\
\text { municipio } \\
\mathbf{( \% )}\end{array}$ & Población & $\begin{array}{c}\text { Porcentaje } \\
\mathbf{d e ~ l a ~} \\
\text { población } \\
\mathbf{( \% )}\end{array}$ & $\begin{array}{c}\text { Extensión } \\
\text { territorial } \\
\left.\mathbf{( k m}^{\mathbf{2}}\right)\end{array}$ & $\begin{array}{c}\text { Porcentaje } \\
\mathbf{\text { de }} \\
\text { extensión } \\
\text { territorial } \\
\mathbf{( \% )}\end{array}$ \\
\hline $\begin{array}{l}\text { De 0.74 a 1 (muy } \\
\text { deficiente) }\end{array}$ & 250 & 10.2 & 3893120 & 3.5 & 201785.20 & 10.3 \\
\hline $\begin{array}{l}\text { De 0.58 a 0.73 } \\
\text { (deficiente) }\end{array}$ & 496 & 20.2 & 8836691 & 7.9 & 336915.99 & 17.2 \\
\hline $\begin{array}{l}\text { De 0.42 a 0.57 } \\
\text { (regular) }\end{array}$ & 739 & 30.1 & 17578666 & 15.6 & 506165.57 & 25.9 \\
\hline
\end{tabular}


Tecnología y

Ciencias $\stackrel{\Xi}{\Im}$ Aua
2020, Instituto Mexicano de Tecnología del Agua

Open Access bajo la licencia CC BY-NC-SA 4.0

(https://creativecommons.org/licenses/by-nc-sa/4.0/)

\begin{tabular}{|l|c|c|c|c|c|c|}
\hline $\begin{array}{l}\text { De } 0.24 \text { a 0.41 } \\
\text { (adecuado) }\end{array}$ & 725 & 29.5 & 44741873 & 39.8 & 559802.18 & 28.6 \\
\hline $\begin{array}{l}\text { De 0.01 a 0.23 } \\
\text { (muy adecuado) }\end{array}$ & 246 & 10.0 & 37286188 & 33.2 & 351570.26 & 18.0 \\
\hline Total & 2456 & 100.0 & 112336538 & 100.0 & $\begin{array}{c}1956 \\
239.19\end{array}$ & 100.0 \\
\hline
\end{tabular}

\section{Índice de sequías $\left(I_{s}\right)$}

En México, el Servicio Meteorológico Nacional (SMN) es la dependencia oficial del gobierno encargada de proporcionar información meteorológica (estado del tiempo) y climatológica. Para ello utiliza las redes de observación, como estaciones meteorológicas automáticas, observatorios sinópticos, radares, estaciones de radio sondeo y estaciones receptoras de imágenes de satélite. Como parte de sus actividades de monitoreo, el SMN se encarga de detectar el estado actual y la evolución de la sequía (Conagua-SMN, 2018) mediante el monitor de sequía en México (MSM), que a su vez forma parte del monitor de sequía de América del Norte (NADM, por sus siglas en inglés). El MSM inició su operación en 2002, dentro de las actividades del NADM, pero no fue sino hasta el año 2014 que adquirió su carácter nacional, lo que le permitió emitir mapas de sequía en escala de tiempo diferente a la mensual, siempre basada en la metodología utilizada por el monitor de sequía de Estados Unidos (USDM, 
por sus siglas en inglés) y el NADM. A partir de febrero de 2014, la emisión del MSM fue quincenal.

Estos índices se despliegan en capas, a través de un sistema de información geográfica (SIG); mediante un consenso se determinan las regiones afectadas por sequía, de acuerdo con la escala de intensidades que es común en los tres países (EUA, México y Canadá), que va desde anormalmente seco (D0), sequía moderada (D1), sequía severa (D2) y sequía extrema (D3) hasta sequía excepcional (D4).

En este contexto, con base en los registros históricos del MSM, para cada categoría de sequía y en función de los datos municipales se desarrolló una metodología para elaborar el mapa nacional de peligro por sequía a escala municipal, el cual se describe a continuación de manera resumida. En IMTA (2017), y Ortega-Gaucin, De la Cruz, y Castellano (2018) se detalla ampliamente.

El peligro por sequía a escala municipal se determinó calculando la probabilidad de ocurrencia de sequía (frecuencia relativa), por medio de los registros del MSM, lo que implicó llevar a cabo las siguientes actividades:

- Obtención de los registros históricos de sequía por municipio, en función de su grado de severidad (D0 a D4) de la página oficial del MSM, y elaboración de una base de datos de Excel. Los datos de los registros históricos de sequía se obtuvieron al ingresar a la liga de la página web del MSM (Conagua-SMN, 2018).

- $\quad$ Análisis de la serie de tiempo de los grados de severidad (D0 a D4) del periodo del 31 de enero de 2003 al 31 de marzo de 2017; el total de meses fueron 171. Sin embargo, en dos de éstos no hubo registro de 
ningún dato (31 de agosto de 2003 y 29 de febrero de 2004); por lo tanto, el total de meses analizados fue de 169.

- Obtención de las frecuencias absolutas correspondientes a cada tipo de severidad de sequía (D0 a D4) por municipio, tomando en cuenta aquellos con al menos el $40 \%$ de su territorio afectado por algún grado de sequía. Para el periodo del 28 de febrero de 2014 al 31 de marzo de 2017, los registros se encontraron de manera quincenal, pero dado que el análisis fue mensual, se eliminaron los registros de la primera quincena de este periodo. Para obtener las frecuencias absolutas se llevó a cabo un conteo de cuántos D0 a D4 hubo en los 169 meses registrados. Posteriormente, la base de datos se ordenó de acuerdo con los municipios. - Cálculo de la probabilidad de sequía mediante la envolvente (D0 a D4) y expresión de la misma en porcentaje. La probabilidad de ocurrencia de un evento determinado, es decir, el nivel de certeza que se tiene de que ocurra un suceso es la razón entre el número de veces en que sucedió dicho evento y el número de repeticiones del experimento. A esta razón se le denomina frecuencia relativa.

Para calcular la frecuencia relativa se utilizó la Ecuación 3:

$P(A)=\frac{A}{N}$

donde:

$P$ : probabilidad de ocurrencia.

$A$ : número de meses con sequía.

$N$ : número total de registros en el periodo. 
Los valores de la probabilidad oscilan en un rango cerrado de [0, 1]. Con el objetivo de clasificar estas probabilidades en percentiles con categorías de igual tamaño, tales valores se ajustaron a una distribución normal y el resultado se multiplicó por 100 para expresarlas en porcentaje. Dado que existen cinco categorías de clasificación de la probabilidad de la sequía, cada rango de percentil obedece al $20 \%$ (Figura 2). 
Tecnología y

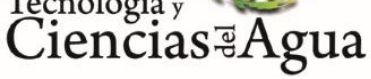

2020, Instituto Mexicano de Tecnología del Agua

Open Access bajo la licencia CC BY-NC-SA 4.0

(https://creativecommons.org/licenses/by-nc-sa/4.0/)

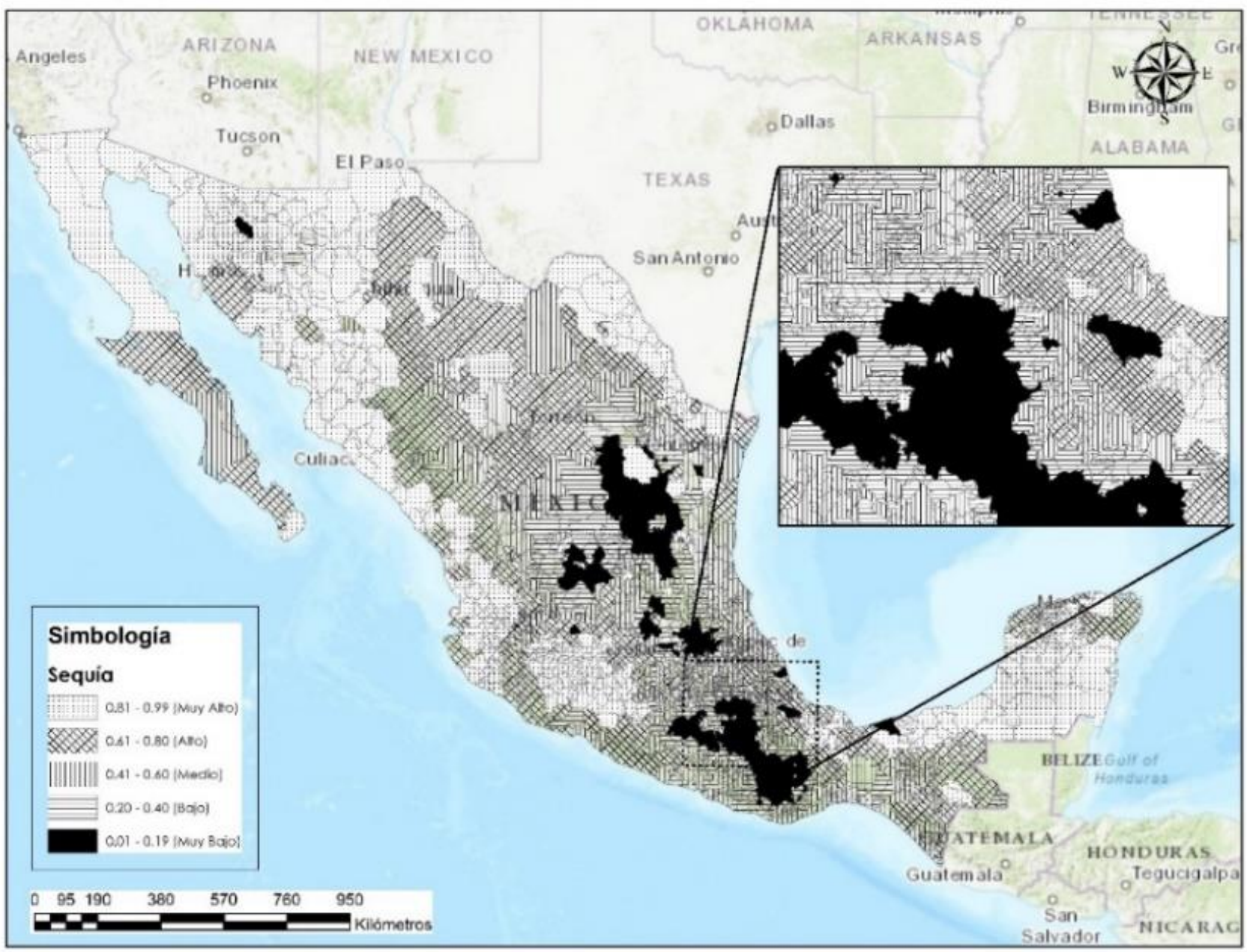

Figura 2. Mapa del índice de sequías por municipio en la república mexicana $\left(\boldsymbol{I}_{s}\right)$.

El estado que presenta mayor número de municipios con afectación es Michoacán, debido a que el $70 \%$ tiene una sequía muy alta; además de los estados del norte, como Baja California, Sonora, Coahuila, y del sureste los estados de Campeche, Quintana Roo y Yucatán. El estado con 
Tecnología y

Ciencias $₫$ Agua
2020, Instituto Mexicano de Tecnología del Agua

Open Access bajo la licencia CC BY-NC-SA 4.0

(https://creativecommons.org/licenses/by-nc-sa/4.0/)

mayor número de municipios de sequía alta es Veracruz, con 71 municipios.

La entidad que tiene el mayor número de municipios en el rango medio, bajo y muy bajo es Oaxaca, con 116 municipios en nivel medio, 108 municipios en nivel bajo y 323 municipios en nivel muy bajo, es decir, el $96 \%$ del total de los municipios presenta algún grado de sequía.

La Tabla 2 expone una disgregación de rangos de sequía por número de municipios, población y superficie; destaca que $17.9 \%$ de los municipios en el que habita $22.3 \%$ de la población está en una zona con muy alta probabilidad de sequía.

Tabla 2. Rangos del índice de sequías $\left(\boldsymbol{I}_{s}\right)$.

\begin{tabular}{|l|c|c|c|c|c|c|}
\hline \multicolumn{1}{|c|}{ Rangos } & $\begin{array}{c}\text { Número de } \\
\text { municipios }\end{array}$ & $\begin{array}{c}\text { Porcentaje } \\
\text { de } \\
\text { municipio } \\
\mathbf{( \% )}\end{array}$ & Población & $\begin{array}{c}\text { Porcentaje } \\
\text { de la } \\
\text { población } \\
\mathbf{( \% )}\end{array}$ & $\begin{array}{c}\text { Extensión } \\
\text { territorial } \\
\left(\mathbf{k m}^{\mathbf{2}}\right)\end{array}$ & $\begin{array}{c}\text { Porcentaje } \\
\text { de } \\
\text { extensión } \\
\text { territorial } \\
\mathbf{( \% )}\end{array}$ \\
\hline $\begin{array}{l}\text { De 0.81 a } \\
0.99 \text { (muy } \\
\text { alto) }\end{array}$ & 440 & 17.9 & 25052980 & 22.3 & 767127.31 & 39.2 \\
\hline $\begin{array}{l}\text { De 0.61 a } \\
0.80 \text { (alto) }\end{array}$ & 472 & 19.2 & 36412593 & 32.4 & 540977.69 & 27.7 \\
\hline $\begin{array}{l}\text { De 0.41 a } \\
0.60 \text { (medio) }\end{array}$ & 587 & 23.9 & 27382986 & 24.4 & 325414.05 & 16.6 \\
\hline $\begin{array}{l}\text { De 0.20 a } \\
0.40 \text { (bajo) }\end{array}$ & 460 & 18.7 & 15959801 & 14.2 & 197958.23 & 10.1 \\
\hline $\begin{array}{l}\text { De 0.01 a } \\
0.19 \text { (muy } \\
\text { bajo) }\end{array}$ & 497 & 20.2 & 7528178 & 6.7 & 124761.92 & 6.4 \\
\hline Total & 2456 & 100.0 & 112336538 & 100.0 & 1956239.19 & 100.0 \\
\hline
\end{tabular}




\section{Índice de abatimiento de aguas subterráneas $\left(I_{\text {aas }}\right)$}

El agua subterránea aún se trata como un recurso invisible e inagotable, cuando en realidad los sistemas acuíferos se han llevado a un uso intensivo nunca antes visto, lo que ocasiona problemas locales (hundimiento del terreno) y regionales (intercepción de flujos regionales, que afectan unidades acuíferas interconectadas, reducción en las salidas naturales de descarga), con afectaciones irreversibles (Custodio, 2000).

En México hace falta entender la dinámica del agua del subsuelo, mucho más lenta que la del agua superficial. El monitoreo de la variación del nivel freático y piezométrico en acuíferos es limitado en espacio (variación sistemática en los puntos de medición, ausencia de un arreglo espacial, de las redes de medición) y tiempo (variación en distintas épocas del año, falta de continuidad a largo tiempo). Desde la década de 1970 se han realizado esfuerzos por establecer redes de monitoreo en acuíferos libres, semiconfinados y confinados del país, pero persisten vacíos de datos en el monitoreo; así, la percepción remota es una tecnología que poco a poco se ha integrado en las hidrociencias, ofreciendo la ventaja de un monitoreo que abarca espacialmente zonas de difícil acceso, con riqueza en información sistemática y continua.

El proyecto Gravity Recovery and Climate Experiment (GRACE, por sus siglas en inglés) permite estimar los volúmenes de agua subterránea 
a partir de las variaciones temporales del campo de gravedad. GRACE es un proyecto americano-alemán llevado a cabo por la Administración Nacional de la Aeronáutica y del Espacio Estadounidense (NASA, por sus siglas en inglés) y el Centro Aeroespacial Alemán (DLR, por sus siglas en alemán, de Deutsches Zentrum für Luft- und Raumfahrt), que consta de dos satélites gemelos que orbitan a $450 \mathrm{~km}$ de altura, separados $220 \mathrm{~km}$ entre ellos. La resolución espacial de la información es unos 200000 km², aplicable en cuencas hidrológicas (Wahr, Zlotnicki, \& Velicogna, 2004; Scalon, Longuevergne, \& Long, 2012). GRACE genera información temporal del campo gravitacional con una periodicidad de 10 días a un mes. La información de la serie L2 de GRACE está disponible en http://www2.csr.utexas.edu/grace/RL06.html.

El geoide o la densidad de masa terrestre (Wahr, Zlotnick, \& Valicogna, 1998) se representa en forma de series finitas de armónicos esféricos, con los cuales se calcula la variación total del almacenamiento de agua (VTAA), expresada en unidades de agua equivalente. EI VTAA integra los cambios del almacenamiento del hielo-nieve (CAHN), agua superficial (CAAS), agua en suelo (CAS) y subsuelo (CASUB); este último se calcula con la Ecuación 4:

$C A S U B=V T A A-C A H N-C A A S-C A S$

La novedad de GRACE es el registro de la variación temporal del campo gravitacional, traducido en VTAA en forma global.

El objetivo de utilizar los datos de GRACE y reducirlos para obtener CASUB radica en la obtención promedio del abatimiento-recuperación en 
la república mexicana, información que se utiliza como índice de agua subterránea en el cálculo de los índices municipales de seguridad hídrica.

Aplicando la metodología estándar de los datos GRACE, de la serie L2 se obtuvieron los registros mensuales de VTAA para el periodo de 2002 a 2015, y aplicando la Ecuación 4 se obtuvo el valor de CASUB. La información de las componentes CAHN, CAAS y CAS se procesó de las bases de datos de resolución de $1^{\circ}$ terrestre, con periodo mensual, desde 2002 hasta 2015.

Cabe destacar que los cambios de masa donde se observa mayor variación corresponden a los ciclos estacionales inviemo-verano y primavera-otoño, es decir, existe un fenómeno de transición estacional. En la época de invierno suceden los mayores abatimientos y en verano las recuperaciones, relacionadas con el efecto de la precipitación. Este fenómeno es típico de zonas áridas y semiáridas. En la época de primavera-otoño existe un proceso intermedio, donde se recupera o abate el valor total de almacenamiento de agua.

En forma general, puede identificarse que en el norte del país se mantiene un valor total del almacenamiento del agua entre estable y abatimiento. Al centro del país, dominado por los organismos de cuenca Lerma-Santiago Pacífico y Golfo Norte, Aguas del Valle de México, Balsas, Pacífico Sur, Golfo Centro y Frontera Sur están las mayores variaciones de masa de agua; en la península de Yucatán tiende a ser estable, debiendo tomarse esto último con reserva, pues los procesos locales son los dominantes y se ocultan al analizarse con los armónicos esféricos de órdenes y grados menores. 
En valor promedio de la CASUB para el mes de julio de los años 2002 a 2015 muestra que la infiltración y la recarga se extienden hacia casi toda la zona costera del Golfo de México, incluyendo la península de Yucatán, y continúan hacia la porción central de la república mexicana hacia la cuenca del valle de México y la cabecera de la cuenca del río Pánuco. En las costas del océano Pacífico, durante el mes de julio se observa un valor de recarga e infiltración, principalmente para la zona media de la península de Baja California, hacia la zona del desierto de Vizcaíno.

En el mes de agosto prevalecen y se incrementan las condiciones existentes en julio, pero se extienden hacia las zonas de la cuenca del río Balsas, las cuencas costeras del Pacífico Norte, entre Hermosillo y Mazatlán, la cabecera de la cuenca del río Lerma y la porción oriental de cuencas centrales.

Octubre es semejante en la distribución a septiembre, pero se muestra más intenso hacia la porción central de la república mexicana, principalmente hacia las cabeceras de las cuencas del Valle de México, río Lerma, Pánuco, Balsas y costas de Veracruz (Xalapa), así como en la porción de la península de Yucatán y costas de Oaxaca.

Al terminar la temporada de lluvias se manifiestan, de igual manera, los efectos de almacenamiento de agua subterránea en noviembre, con una disminución o atenuación progresiva de dichas zonas para diciembre.

El almacenamiento de agua subterránea se calculó mes a mes, en el periodo 2002-2015; después se estimó el valor promedio mensual y anual. El resultado es el almacenamiento de agua subterránea promedio en un periodo de 13 años, por lo que el almacenamiento de agua 
subterránea, en caso de ser positivo, se considera como recuperación, y en el caso de presentar valores negativos corresponde a abatimientos. Para obtener los valores de almacenamiento por municipio se llevó a cabo una interpolación con los resultados de la Figura 3 y la división municipal; después se aplicó la normalización.

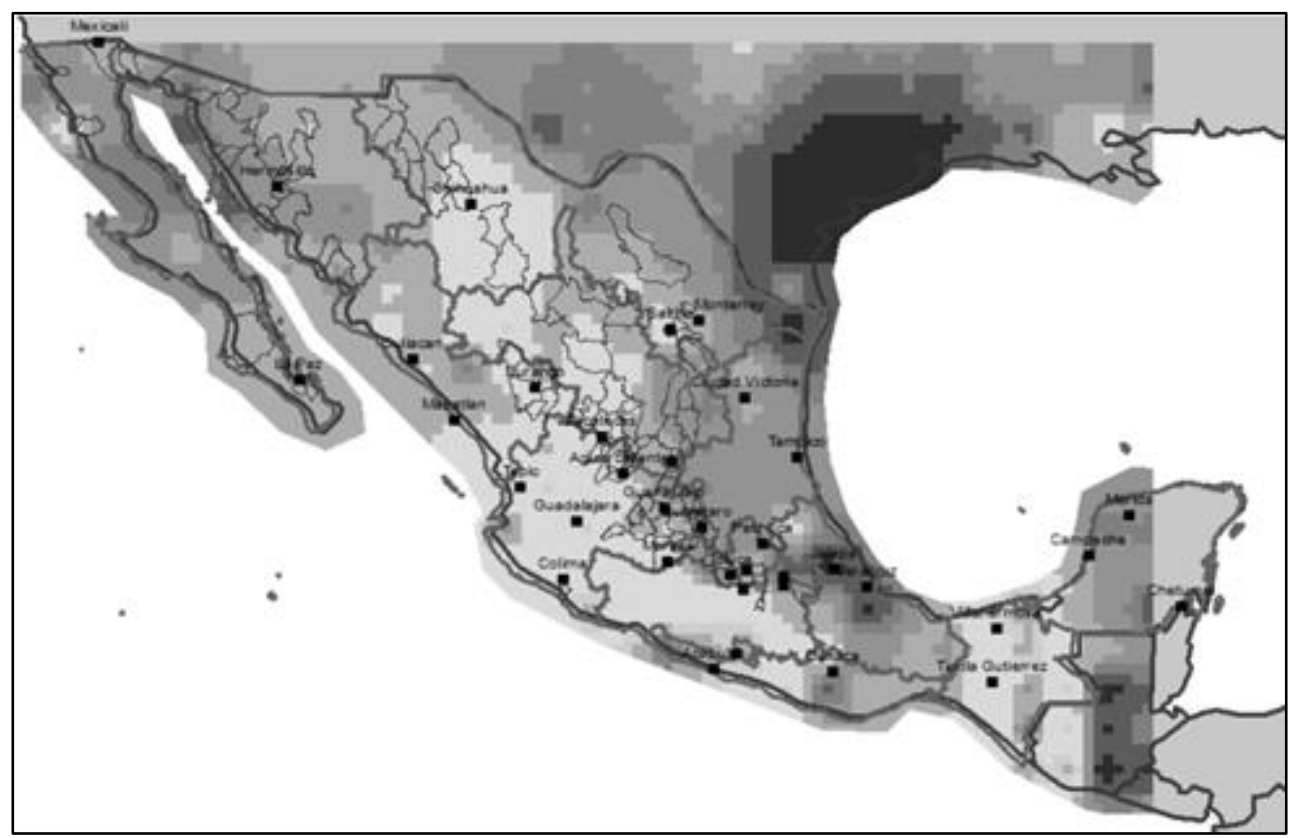

Figura 3. Valor promedio de CASUB (cambio de almacenamiento en el subsuelo) en la república mexicana, periodo 2002-2015.

El método de normalización aplicado a los valores de abatimiento/recuperación, para escala de 0 a 1 es el mín-máx, como se muestra en OECD (2008). La clasificación de datos para determinar la categorización correcta de los valores, de acuerdo con las rupturas o huecos que existen naturalmente entre éstos fue maximizando la varianza entre clases y minimizando la varianza dentro de las clases (Jenks, 1967) 
Tecnología y

Ciencias $₫$ Agua
2020, Instituto Mexicano de Tecnología del Agua

Open Access bajo la licencia CC BY-NC-SA 4.0

(https://creativecommons.org/licenses/by-nc-sa/4.0/)

con el programa QGis; los resultados gráficos en mapa de abatimientos se plasman en la Figura 4.

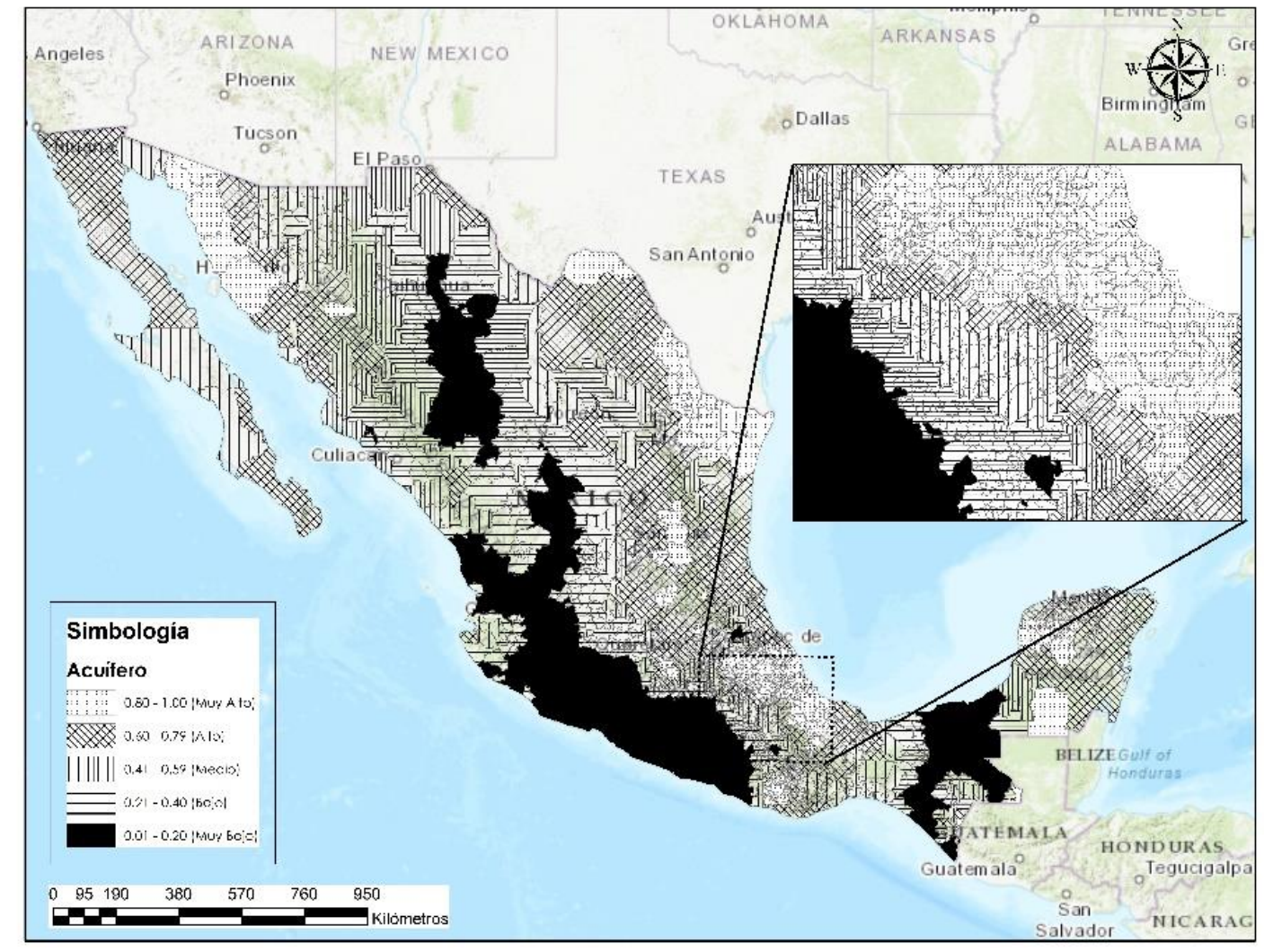

Figura 4. Mapa de índice por abatimiento de aguas subterráneas $\boldsymbol{I}_{\boldsymbol{a a s}}$.

El estado de Jalisco presenta el mayor número de municipios con abatimiento muy alto de aguas subterráneas (85); representan el 68\% del total de sus municipios. En un rango alto y medio de abatimiento está Oaxaca, con 115 municipios en cada caso, aunque también cuenta con 200 en una condición aceptable. El estado de Veracruz tiene 102 de sus municipios sin abatimientos (48\%). 
Tecnología y

Ciencias $₫$ Agua
2020, Instituto Mexicano de Tecnología del Agua

Open Access bajo la licencia CC BY-NC-SA 4.0

(https://creativecommons.org/licenses/by-nc-sa/4.0/)

En México existen 519 municipios que presentan abatimientos muy altos (21\%, con una población de 22520521 habitantes y un área de $369377.33 \mathrm{~km}^{2}$ ). Con abatimientos altos se tienen 431 municipios, en los que habitan 26504321 personas, en un área de 356627.33 km². Aquellos en un rango medio de abatimiento son 397. Por último, 1 109, en los que habitan 39300516 personas tienen muy bajo abatimiento (45.15\% del total de municipios) (ver Tabla 3).

Tabla 3. Disgregación de abatimiento de aguas subterráneas $\boldsymbol{I}_{\text {aas }}$ por municipios.

\begin{tabular}{|c|c|c|c|c|c|c|}
\hline Rangos & Municipios & $\begin{array}{c}\text { Porcentaje } \\
\text { de municipio } \\
(\%)\end{array}$ & Población & $\begin{array}{c}\text { Porcentaje } \\
\text { de la } \\
\text { población } \\
(\%)\end{array}$ & Área $\left(\mathbf{k m}^{2}\right)$ & $\begin{array}{c}\text { Porcentaje } \\
\text { de extensión } \\
\text { territorial } \\
(\%)\end{array}$ \\
\hline $\begin{array}{l}\text { De } 0.80 \text { a } 0.99 \\
\text { (muy alto) }\end{array}$ & 519 & 21.1 & 22520521 & 20.0 & 369377.33 & 19 \\
\hline $\begin{array}{l}\text { De } 0.60 \text { a } 0.79 \\
\text { (alto) }\end{array}$ & 431 & 17.5 & 26504321 & 23.6 & 356627.33 & 18 \\
\hline $\begin{array}{l}\text { De } 0.41 \text { a } 0.59 \\
\text { (medio) }\end{array}$ & 397 & 16.2 & 24011180 & 21.4 & 402113.93 & 21 \\
\hline $\begin{array}{l}\text { De } 0.21 \text { a } 0.40 \\
\text { (bajo) }\end{array}$ & 717 & 29.2 & 27987330 & 24.9 & 608944.16 & 31 \\
\hline $\begin{array}{l}\text { De } 0.00 \text { a } 0.20 \\
\text { (muy bajo) }\end{array}$ & 392 & 16.0 & 11313186 & 10.1 & 219176.45 & 11 \\
\hline Total & 2456 & 100.0 & 112336538 & 100.0 & 1956239.19 & 100 \\
\hline
\end{tabular}

\section{Índice de inundaciones y fenómenos meteorológicos extremos $\left(I_{i f m e}\right)$}


El Sistema de Nacional de Información del Agua (Sina), de la Conagua publicó información municipal acerca de las declaratorias de emergencia emitidas de 2000 a 2016 por fenómenos hidrometeorológicos. En el país existen procedimientos de emisión de declaratorias ante tales fenómenos, en los que participa el Cenapred. La información extraída contiene datos de ciclones, Iluvia, inundaciones, bajas temperaturas, nieve, granizada y tornados (Conagua-Sina, 2016).

A partir de información publicada del periodo indicado, se tomó el número total de declaratorias de emergencia por municipio, incluyendo ciclones, Iluvias intensas, inundaciones, bajas temperaturas, fuertes vientos, nevada-helada y tornados. Este número se dividió entre los días de un año para obtener un valor anual de declaratorias por municipio. La información se normalizó a través de la función de distribución normal, donde se obtuvo la media $\mu$ y la varianza de la distribución $\sigma^{2}$ de la serie de valores anuales de declaratorias por municipio.

La clasificación de datos para determinar la mejor categorización de los valores con base en las rupturas o huecos que existen naturalmente entre los datos, se logra maximizando la varianza entre clases y minimizando la varianza dentro de las clases (Jenks, 1967) mediante el programa QGis de acuerdo con la Ecuación 5:

$F(x)=\int_{-\infty}^{x} \frac{1}{\sigma \sqrt{2 \pi}} e^{-\frac{(x-\mu)^{2}}{2 \sigma} d x}$ para $-\infty \leq x \leq \infty$ 
Ciencias

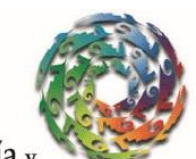

2020, Instituto Mexicano de Tecnología del Agua

Open Access bajo la licencia CC BY-NC-SA 4.0

(https://creativecommons.org/licenses/by-nc-sa/4.0/)

La Figura 5 presenta el mapa de peligro por fenómenos hidrometeorológicos. Se observa que el estado con mayor recurrencia (muy alto) de fenómenos meteorológicos extremos es Veracruz, con un $70 \%$ del total de sus municipios (149). Con nivel alto, medio, bajo y muy bajo se encuentra Oaxaca; $96 \%$ de total de sus municipios ha tenido estragos por algún evento meteorológico extremo.

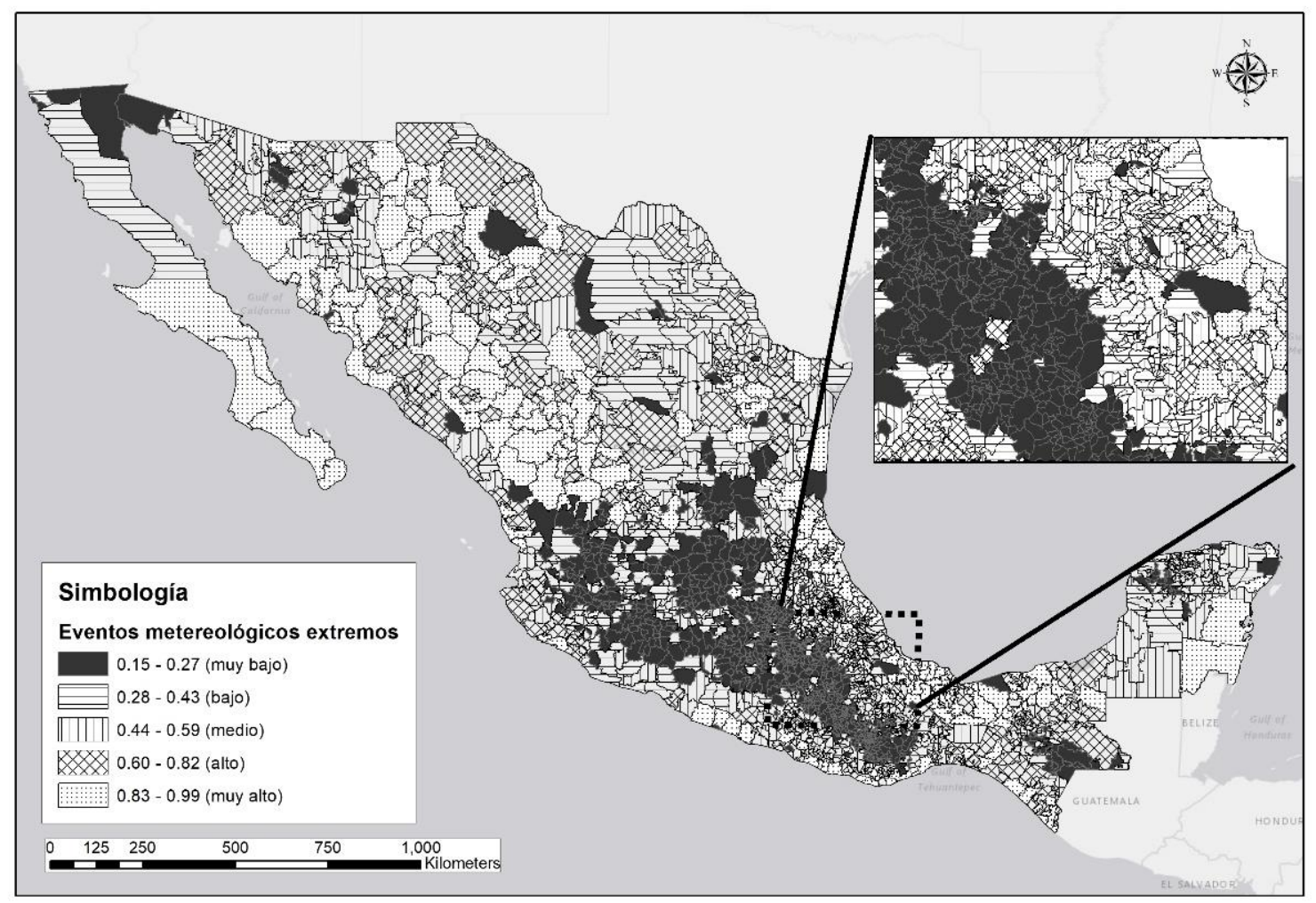

Figura 5. Mapa de índice de inundaciones y fenómenos meteorológicos extremos $\boldsymbol{I}_{\text {ifme }}$. 
Tecnología y

Ciencias $₫$ Agua
2020, Instituto Mexicano de Tecnología del Agua

Open Access bajo la licencia CC BY-NC-SA 4.0

(https://creativecommons.org/licenses/by-nc-sa/4.0/)

La población afectada en el rango muy alto es de 22676963.00 personas, ubicadas en un área de 529637.77 km², en 330 municipios afectados. En el rango alto están 359 municipios, con 14637033 habitantes, ubicados en $429908.29 \mathrm{~km}^{2}$. El rango bajo y muy bajo integra 1426 municipios, con 64642429 habitantes y una frecuencia mínima de estos eventos (Tabla 4).

Tabla 4. Clasificación municipal de rangos de peligro de inundaciones y fenómenos meteorológicos extremos $\boldsymbol{I}_{\text {ifme }}$.

\begin{tabular}{|l|c|c|c|c|c|c|}
\hline \multicolumn{1}{|c|}{ Rangos } & Municipios & $\begin{array}{c}\text { Porcentaje } \\
\text { de } \\
\text { municipio } \\
\mathbf{( \% )}\end{array}$ & Población & $\begin{array}{c}\text { Porcentaje } \\
\text { de la } \\
\text { población } \\
\mathbf{( \% )}\end{array}$ & Área (km $\mathbf{~}^{\mathbf{2}}$ & $\begin{array}{c}\text { Porcentaje } \\
\text { de extensión } \\
\text { territorial } \\
\mathbf{( \% )}\end{array}$ \\
\hline $\begin{array}{l}\text { De } 0.83 \text { a } 0.99 \\
\text { (muy alto) }\end{array}$ & 330 & 13.4 & 22676963 & 20.2 & 529637.77 & 27.1 \\
\hline $\begin{array}{l}\text { De } 0.60 \text { a } 0.82 \\
\text { (alto) }\end{array}$ & 359 & 14.6 & 14637033 & 13.0 & 429908.29 & 22.0 \\
\hline $\begin{array}{l}\text { De } 0.44 \text { a } 0.59 \\
\text { (medio) }\end{array}$ & 341 & 13.9 & 10380113 & 9.2 & 277972.60 & 14.2 \\
\hline $\begin{array}{l}\text { De } 0.28 \text { a } 0.43 \\
\text { (bajo) }\end{array}$ & 454 & 18.5 & 21381351 & 19.0 & 381352.91 & 19.5 \\
\hline $\begin{array}{l}\text { De } 0.15 \text { a } 0.27 \\
\text { (muy bajo) }\end{array}$ & 972 & 39.6 & 43261078 & 38.5 & 337367.63 & 17.2 \\
\hline Total & 2456 & 100.0 & 112336538 & 100.0 & 1956239.19 & 100.0 \\
\hline
\end{tabular}

\section{Índice de peligro $\left(I_{P}\right)$}


Teçnología y

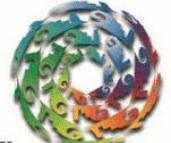

Ciencias $₫$ Agua
2020, Instituto Mexicano de Tecnología del Agua

Open Access bajo la licencia CC BY-NC-SA 4.0

(https://creativecommons.org/licenses/by-nc-sa/4.0/)

Para integrar todos estos parámetros en un índice, se normalizaron con valores que van de 0 a 1 ; así, el índice de peligro $I_{p}$ se calcula con la Ecuación 6. El mapa de índice de peligro resultante de la integración de estos cuatro parámetros se presenta en la Figura 6.

$I_{P}=I_{\text {fasas }}+I_{s}+I_{\text {aas }}+I_{\text {ifme }}$

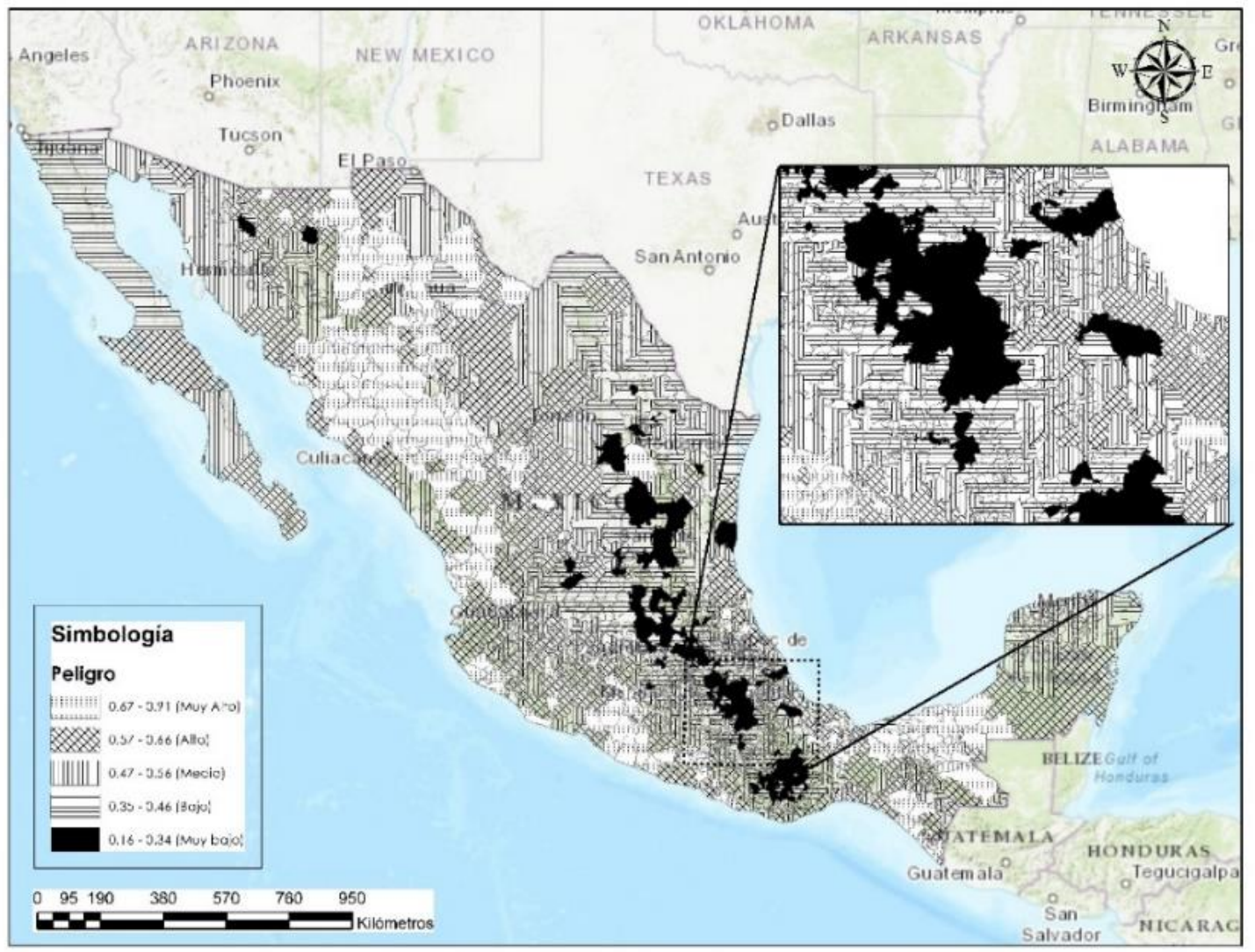

Figura 6. Índice de peligro municipal $\boldsymbol{I}_{\boldsymbol{P}}$. 


\section{Índice de vulnerabilidad social $\left(I_{v s}\right)$}

El Centro Nacional de Prevención de Desastres (Cenapred) define la vulnerabilidad social como: "el conjunto de características sociales y económicas de la población que limita la capacidad de desarrollo de la sociedad; en conjunto con la capacidad de prevención y respuesta de la misma frente a un fenómeno y la percepción local del riesgo de la población" (Cenapred-AR, 2014).

Para el cálculo del índice de vulnerabilidad social se eligieron indicadores que identifican las principales características de la población, su capacidad de organización y elementos indispensables para la atención de una emergencia. De la información disponible en el Instituto Nacional de Estadística, Geografía e Informática (INEGI), obtenida del censo del año 2010 y con base en la metodología propuesta por Cenapred, se calculan indicadores como:

- Grado promedio de escolaridad (GPES).

- Proporción de médicos por cada 1000 habitantes $(P M)$.

- Tasa de mortalidad infantil (TMI).

- Población no derechohabiente (PND).

- Analfabetismo $(A)$.

- Viviendas sin servicio de agua potable (TVNDAE).

- Viviendas sin servicio de drenaje (TVND). 
- Viviendas sin servicios de electricidad (TVNDE).

- Viviendas con piso de tierra (TVPH).

- Población económicamente activa (PEA).

- Razón de dependencia (RD).

- Tasa de desempleo abierto (TDA).

- Población de habla indígena $(P I)$.

El índice de vulnerabilidad social $\left(I_{v s}\right)$ por cada municipio, una vez normalizados los datos, se calcula con la Ecuación 7:

$I_{V S}=\frac{\sum_{i=1}^{n} v_{i}}{n}$

donde:

Ivs: índice de vulnerabilidad municipal .

$v_{i}$ : valor de cada uno de los indicadores de socioeconómicos de la vulnerabilidad social: GPES, PM, TMI, PND, A, TVNDAE, TVND, TVNDE, $T V P H, P E A, R D, T D A, P I$.

$n$ : número de indicadores.

De la Ecuación 7 se clasificaron los resultados por municipio para generar un mapa de índice de vulnerabilidad social. 


\section{Índice de riesgo e índice de seguridad hídrica}

La Figura 7 indica la metodología para calcular los mapas de índice de riesgo $\left(I_{R}\right)$ e índice de seguridad hídrica $\left(I_{S H}\right)$, donde es necesario tener los mapas de índice de peligro $\left(I_{P}\right)$ e índice de vulnerabilidad social $\left(I_{v s}\right)$. 
- Indice de falta y acceso a los servicios de agua y saneamiento

- Indice de sequias

- Indice de abatimiento de aguas subterráneas

- Indice de inundaciones y fenómenos hidrometeorológicos extremos
- Grado promedio de escolaridad (GEPS)

- Proporción de médicos por cada 1000 habitantes $(P M)$

- Tasa mortalidad infantil (TMI)

- Población no derechohabiente (PND)

- Analfabetismo $(A)$

- Viviendas sin servicio de agua potable (TVNDAE)

- Viviendas sin servicio de drenaje (TVND)

- Viviendas sin servicio de electricidad (TVNDE)

- Viviendas con piso de tierra (TVPH)

- Población económicamente activa (PEA)

- Razón de dependencia $(R D)$

- Tasa de desempleo abierto (TDA)

- Población de habla indigena $(P I)$

Suma de datos y normalización

Mapa de indice de peligro $I_{p}$
Suma de datos y normalización

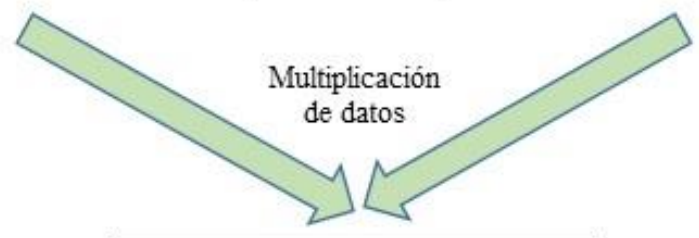

Mapa de indice de riesgo $I_{R}$

$$
\left\lceil I_{S H}=1-I_{R}\right.
$$

Mapa de indice de seguridad hidrica

$$
I_{S H}
$$

Figura 7. Esquema de la metodología probabilística propuesta de seguridad hídrica. 
El riesgo se define como la contingencia o la proximidad al daño, y puede entenderse como la posibilidad de tener pérdidas significativas o resultados adversos. Una definición alternativa es la probabilidad de que ocurra un evento determinado (Ilamado peligro), teniendo en cuenta las características y circunstancias de una ciudad que la hacen susceptible a los efectos que sufriría en términos de salud humana, medio ambiente y actividades económicas (vulnerabilidad).

Un nivel idéntico de peligro afecta a los diferentes estratos de la población del país de diferentes maneras, en especial los municipios; por lo tanto, para definir el índice de riesgo para cada municipio se retoma la metodología propuesta por Liu, Tan, Fang y Lok (2012), y por la Federal Emergency Management Agency (FEMA) de EUA, la cual propone un enfoque que integra tres parámetros: peligro $\left(I_{P}\right)$, vulnerabilidad $\left(I_{v s}\right)$ e impacto $\left(I_{i m}\right)$, es decir, el número de personas que estarían afectadas por la presencia de un evento de peligro, de acuerdo con la Ecuación 8:

$I_{R}=I_{P} \times I_{v s} \times I_{i m}$

Para realizar la categorización de cada mapa de peligro y vulnerabilidad se utilizó el software QGIS, que incorpora el algoritmo de Jenks, que trata de encontrar, mediante un proceso iterativo, agrupaciones naturales de los datos para crear clases que minimizan la variabilidad dentro del grupo y maximizan la variabilidad entre grupos, en un intento de crear clases más homogéneas (Jenks, 1967).

La seguridad hídrica está estrechamente relacionada con el concepto de riesgo, éste mide el daño que los procesos biofísicos y 
sociales pueden ocasionar a la población y los ecosistemas. La seguridad hídrica muestra la capacidad de una sociedad para satisfacer sus necesidades básicas de agua; la conservación y el uso sustentable de los ecosistemas acuáticos y terrestres; y la capacidad para producir alimentos sin atentar contra la calidad y cantidad de los recursos hídricos disponibles, los mecanismos y regulaciones sociales para reducir y manejar los conflictos o disputas por el agua.

Para establecer el índice de seguridad hídrica es necesario tomar en cuenta que el índice de riesgo es el complemento a uno del índice de seguridad hídrica (Ecuación 9):

$I_{S H}=1-I_{R}$

donde:

$I_{S H}:$ índice de seguridad.

$I_{R}:$ índice de riesgo.

\section{Resultados}

\section{Índice de peligro $\left(I_{P}\right)$ en el ámbito municipal}


Se generó el índice para todos los municipios del país a partir de los indicadores de peligro obtenidos. Los resultados se agruparon mediante el método de rupturas generales y se presentan en la Figura 6.

El estado con mayor número de municipios en un nivel muy alto de peligro es Chihuahua, con 36 municipios; en el nivel alto está el estado de Veracruz con 73 municipios; en Oaxaca son 101 municipios en nivel medio, 201 en nivel bajo y 188 en nivel muy bajo.

En todo el país, en nivel muy alto de peligro están 205 municipios, que corresponden a una población de 9426917 habitantes, en una superficie de $408686.10 \mathrm{~km}^{2}$; en el nivel alto se ubican 482 municipios, con una población de 20609368 habitantes, en un área de 592809.62 $\mathrm{km}^{2}$. El nivel medio de peligro se integra por 644 municipios, que representan $26.2 \%$ del total, más del $23.2 \%$ del total de la población, en un área de 592809.62 km². En el nivel bajo de peligro está el 28\% (688), que no presenta problemas mayores, con una población del $38 \%$. Por último, el nivel muy bajo está integrado por 437 municipios (Tabla 5).

Tabla 5. Disgregación municipal de índice de peligro $\boldsymbol{I}_{\boldsymbol{P}}$.

\begin{tabular}{|c|c|c|c|c|l|c|}
\hline Rangos & Municipios & $\begin{array}{c}\text { Porcentaje } \\
\text { de municipio } \\
(\%)\end{array}$ & Población & $\begin{array}{c}\text { Porcentaje } \\
\text { de la } \\
\text { población } \\
(\%)\end{array}$ & Área $\left.\mathbf{( k m}^{2}\right)$ & $\begin{array}{c}\text { Porcentaje } \\
\text { de } \\
\text { extensión } \\
\text { territorial } \\
(\%)\end{array}$ \\
\hline
\end{tabular}


Tecnología y

Ciencias $₫$ Agua
2020, Instituto Mexicano de Tecnología del Agua

Open Access bajo la licencia CC BY-NC-SA 4.0

(https://creativecommons.org/licenses/by-nc-sa/4.0/)

\begin{tabular}{|l|c|c|c|c|c|c|}
\hline $\begin{array}{l}\text { De } 0.67 \text { a } 0.91 \\
\text { (muy alto) }\end{array}$ & 205 & 8.3 & 9426917 & 8.4 & 408686.10 & 20.9 \\
\hline $\begin{array}{l}\text { De 0.57 a 0.66 } \\
\text { (alto) }\end{array}$ & 482 & 19.6 & 20609368 & 18.3 & 592809.62 & 30.3 \\
\hline $\begin{array}{l}\text { De 0.47 a 0.56 } \\
\text { (medio) }\end{array}$ & 644 & 26.2 & 26138848 & 23.3 & 489255.19 & 25.0 \\
\hline $\begin{array}{l}\text { De 0.35 a } 0.46 \\
\text { (bajo) }\end{array}$ & 688 & 28.0 & 42582808 & 37.9 & 371680.30 & 19.0 \\
\hline $\begin{array}{l}\text { De 0.16 a 0.34 } \\
\text { (muy bajo) }\end{array}$ & 437 & 17.8 & 13578597 & 12.1 & 93807.99 & 4.8 \\
\hline Total & 2456 & 100.0 & 112336538 & 100.0 & 1956239.19 & 100.0 \\
\hline
\end{tabular}

\section{Índice de vulnerabilidad social $\left(I_{v s}\right)$ en el ámbito municipal}

La Figura 8 ilustra los resultados obtenidos del índice vulnerabilidad social en cada uno de los municipios de México. El agrupamiento de los datos se realizó con el método de rupturas naturales (Jenks, 1967). 
Tecnología y

Ciencias $₫$ Agua
2020, Instituto Mexicano de Tecnología del Agua

Open Access bajo la licencia CC BY-NC-SA 4.0

(https://creativecommons.org/licenses/by-nc-sa/4.0/)

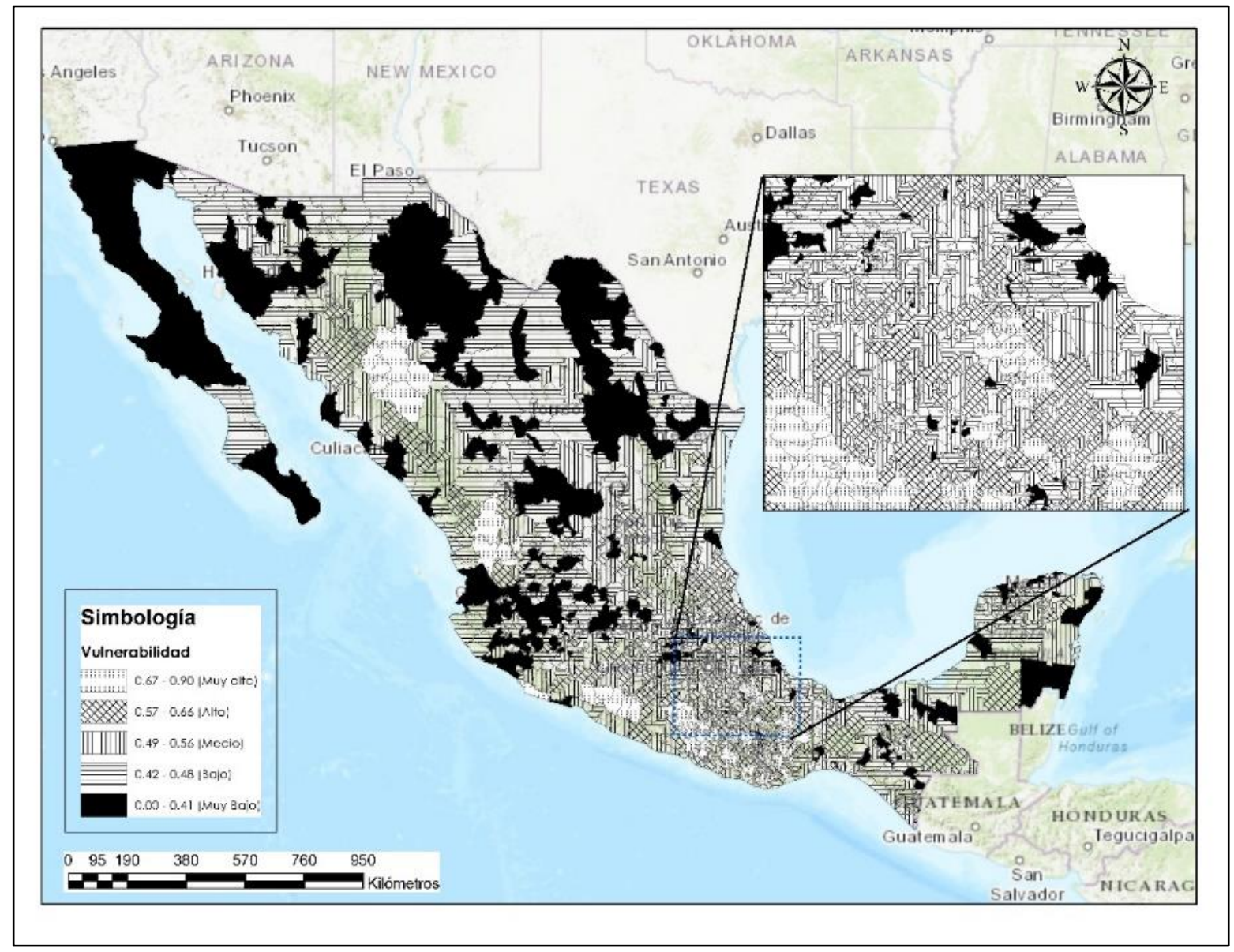

Figura 6. Índice de vulnerabilidad social $\boldsymbol{I}_{\boldsymbol{v}}$ en la república mexicana.

El estado de Oaxaca presenta muy alta vulnerabilidad en 131 municipios, alta en 209 municipios, y media en 72 municipios, es decir, $81 \%$ de total de municipios del estado de Oaxaca tiene vulnerabilidad de media a muy alta.

En el rango de muy alta vulnerabilidad están 220 municipios en el país (Tabla 6), con 2790219 habitantes, en un área de 106979.01 km². En vulnerabilidad alta se encuentran 483 municipios, con 7389613 habitantes, en un área de $221442.24 \mathrm{~km}^{2}$; y en el nivel medio de 
Tecnología y

Ciencias $₫$ Agua
2020, Instituto Mexicano de Tecnología del Agua

Open Access bajo la licencia CC BY-NC-SA 4.0

(https://creativecommons.org/licenses/by-nc-sa/4.0/)

vulnerabilidad están 585 municipios, con una población de 14543384 habitantes.

Tabla 6. Vulnerabilidad social $\boldsymbol{I}_{v s}$ en la república mexicana.

\begin{tabular}{|l|c|c|c|c|c|c|}
\hline \multicolumn{1}{|c|}{ Rangos } & Municipios & $\begin{array}{c}\text { Porcentaje de } \\
\text { municipio } \\
(\%)\end{array}$ & Población & $\begin{array}{c}\text { Porcentaje } \\
\text { de la } \\
\text { población } \\
\mathbf{( \% )}\end{array}$ & Área (km²) & $\begin{array}{c}\text { Porcentaje } \\
\text { de } \\
\text { extensión } \\
\text { territorial } \\
\text { (\%) }\end{array}$ \\
\hline $\begin{array}{l}\text { De 0.67 a 0.90 } \\
\text { (muy alta) }\end{array}$ & 220 & 8.2 & 2722595 & 2.4 & 101432.09 & 5.2 \\
\hline $\begin{array}{l}\text { De 0.57 a 0.66 } \\
\text { (alta) }\end{array}$ & 483 & 18.0 & 7210518 & 6.4 & 209960.34 & 10.7 \\
\hline $\begin{array}{l}\text { De 0.49 a 0.56 } \\
\text { (media) }\end{array}$ & 585 & 21.9 & 14190910 & 12.6 & 377004.06 & 19.3 \\
\hline $\begin{array}{l}\text { De 0.42 a 0.48 } \\
\text { (baja) }\end{array}$ & 694 & 25.9 & 31856037 & 28.4 & 610561.71 & 31.2 \\
\hline $\begin{array}{l}\text { De 0.00 a 0.41 } \\
\text { (muy baja) }\end{array}$ & 474 & 17.7 & 53633884 & 47.7 & 555848.91 & 28.4 \\
\hline $\begin{array}{l}\text { De 0.67 a 0.90 } \\
\text { (muy alta) }\end{array}$ & 220 & 8.2 & 2722595 & 2.4 & 101432.09 & 5.2 \\
\hline Total & 2676 & 100.0 & 112336538 & 100.0 & 1956239.19 & 100.0 \\
\hline
\end{tabular}

Nivel bajo de vulnerabilidad existe en 694 municipios, que presentan una adaptabilidad a un agente perturbador; en este rango se calcula una población de 32647278 habitantes, en un área de 643950.92 $\mathrm{km}^{2}$. El nivel muy bajo lo integran 474 municipios, que corresponden al $48.9 \%$ del total de la población (Figura 8). 
Tecnología y

Ciencias $₫$ Agua
2020, Instituto Mexicano de Tecnología del Agua

Open Access bajo la licencia CC BY-NC-SA 4.0

(https://creativecommons.org/licenses/by-nc-sa/4.0/)

\section{Índice de riesgo $\left(I_{R}\right)$}

En la Figura 7 se presentan los valores obtenidos de índice de riesgo para los municipios de México. La entidad con mayor número de municipios con índice de riesgo muy alto es Guerrero, con 23. En el país, 75 municipios, con una población total de 1914785 habitantes y un área de $121860.86 \mathrm{~km}^{2}$ presentan riesgo muy alto. Por otro lado, 255 municipios en los que se tiene una población de 6408858 habitantes tienen nivel alto de riesgo. Para el nivel bajo y muy bajo se contabilizan 1625 municipios, con el $79 \%$ de la población, los cuales no presentan mayores problemas sobre este índice (Tabla 7). 
Tecnología y

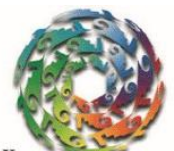

Ciencias $\approx$ Agua
2020, Instituto Mexicano de Tecnología del Agua

Open Access bajo la licencia CC BY-NC-SA 4.0

(https://creativecommons.org/licenses/by-nc-sa/4.0/)

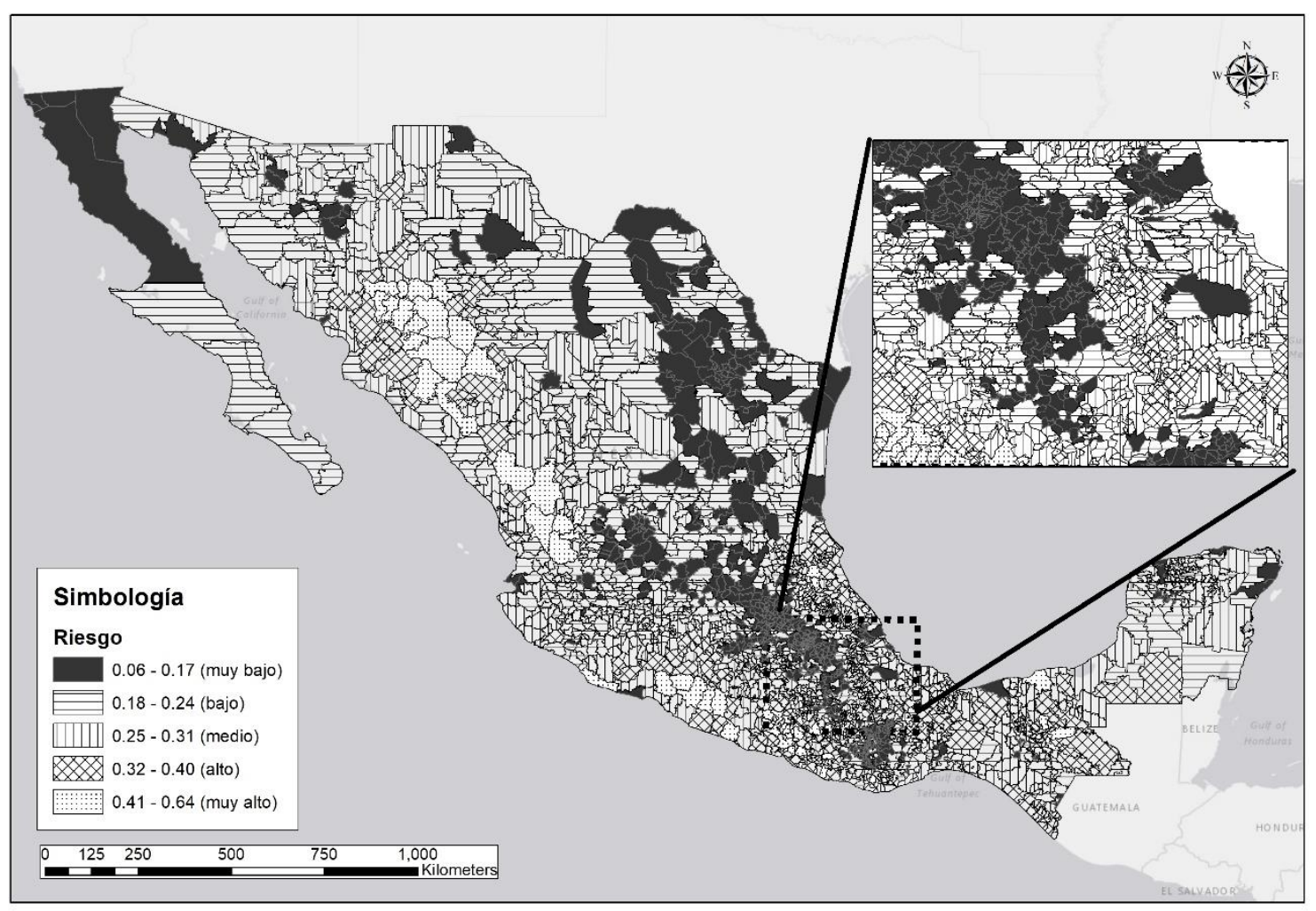

Figura 7. Índice de riesgo $I_{R}$ a nivel municipal.

Tabla 7. Niveles de riesgo $I_{R}$ por rangos.

\begin{tabular}{|l|c|c|c|c|c|c|}
\hline \multicolumn{1}{|c|}{ Rangos } & Municipios & $\begin{array}{c}\text { Porcentaje } \\
\mathbf{d e} \\
\text { municipio } \\
\mathbf{( \% )}\end{array}$ & Población & $\begin{array}{c}\text { Porcentaje } \\
\text { de la } \\
\text { población } \\
\mathbf{( \% )}\end{array}$ & Área (km $\mathbf{~}^{\mathbf{2}}$ & $\begin{array}{c}\text { Porcentaje de } \\
\text { extensión } \\
\text { territorial (\%) }\end{array}$ \\
\hline $\begin{array}{l}\text { De } 0.41 \mathrm{a} \\
0.64 \text { (muy } \\
\text { alto) }\end{array}$ & 75 & 3.1 & 1914785 & 1.7 & 121860.86 & 6.2 \\
\hline $\begin{array}{l}\text { De } 0.32 \mathrm{a} \\
0.40 \text { (alto) }\end{array}$ & 255 & 10.4 & 6408858 & 5.7 & 214231.76 & 11.0 \\
\hline
\end{tabular}


Tecnología y

Ciencias $₫$ Agua
2020, Instituto Mexicano de Tecnología del Agua

Open Access bajo la licencia CC BY-NC-SA 4.0

(https://creativecommons.org/licenses/by-nc-sa/4.0/)

\begin{tabular}{|l|c|c|c|c|c|c|}
\hline $\begin{array}{l}\text { De } 0.25 a \\
0.31 \\
\text { (medio) }\end{array}$ & 501 & 20.4 & 15149331 & 13.5 & 474045.76 & 24.2 \\
\hline $\begin{array}{l}\text { De 0.18 a } \\
0.24 \text { (bajo) }\end{array}$ & 888 & 36.2 & 33873496 & 30.2 & 760732.04 & 38.9 \\
\hline $\begin{array}{l}\text { De 0.06 a } \\
\begin{array}{l}\text { (17 (muy } \\
\text { bajo) }\end{array}\end{array}$ & 737 & 30.0 & 54990068 & 49.0 & 385368.78 & 19.7 \\
\hline Total & 2456 & 100.0 & 112336538 & 100.0 & 1956239.19 & 100.0 \\
\hline
\end{tabular}

\section{Índice de seguridad hídrica municipal $I_{S H}$}

La Figura 8 indica los resultados gráficos de seguridad hídrica obtenidos por municipio, con valores que varían entre 0.35 y 0.94 , agrupados mediante el método de rupturas naturales (Tabla 8 ). 
Tecnología y

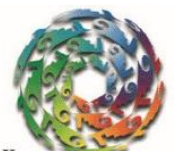

Ciencias 고의
2020, Instituto Mexicano de Tecnología del Agua

Open Access bajo la licencia CC BY-NC-SA 4.0

(https://creativecommons.org/licenses/by-nc-sa/4.0/)

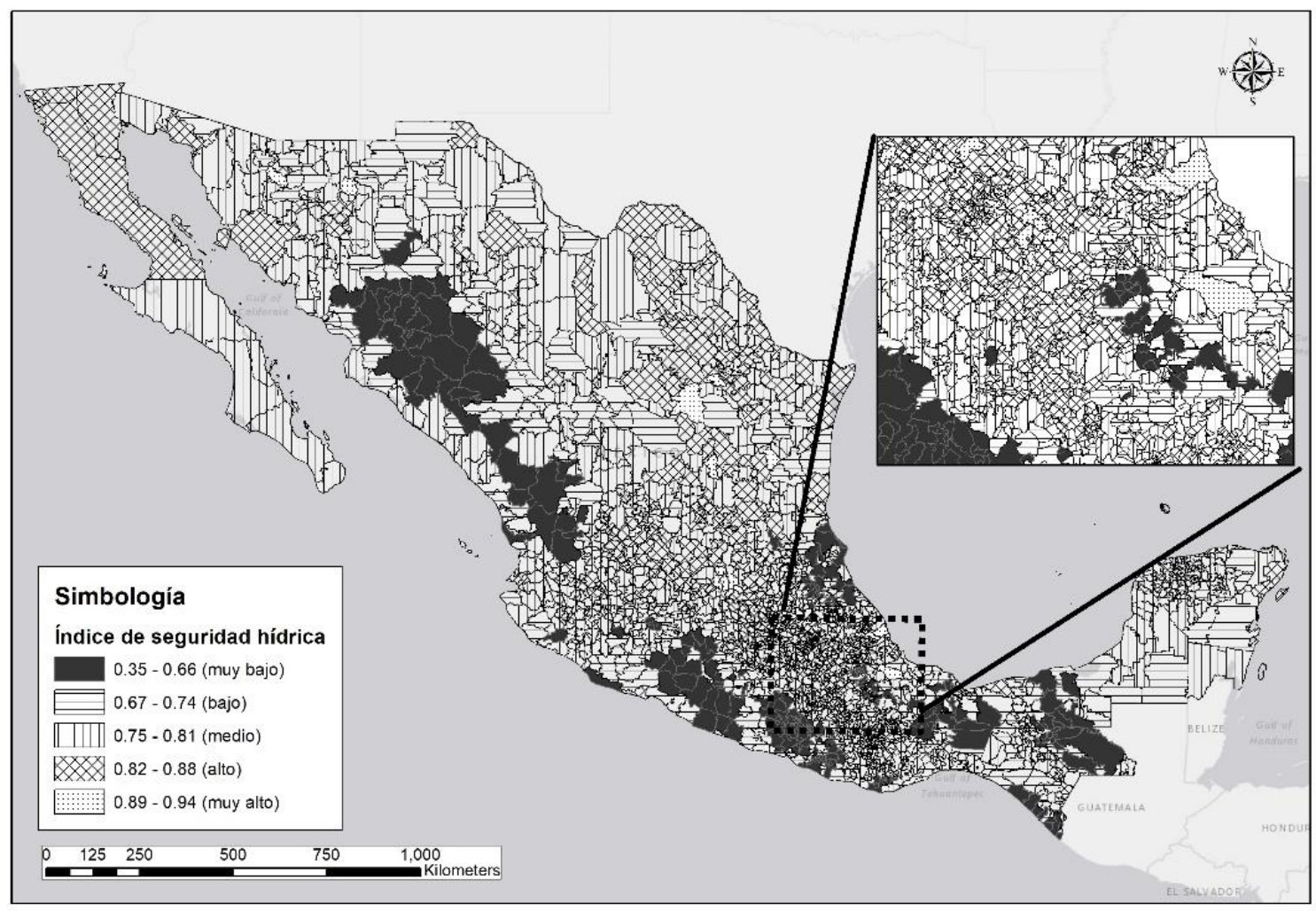

Figura 8. Índice de seguridad hídrica $I_{S H}$ municipal.

Tabla 8. Disgregación del índice de seguridad hídrica $\boldsymbol{I}_{S H}$ en el ámbito municipal.

\begin{tabular}{|l|c|c|c|c|c|c|}
\hline Rangos & Municipios & $\begin{array}{c}\text { Porcentaje } \\
\text { de } \\
\text { municipio } \\
(\%)\end{array}$ & Población & $\begin{array}{c}\text { Porcentaje } \\
\text { de la } \\
\text { población } \\
\mathbf{( \% )}\end{array}$ & Área (km²) & $\begin{array}{c}\text { Porcentaje de } \\
\text { extensión } \\
\text { territorial (\%) }\end{array}$ \\
\hline $\begin{array}{l}\text { De } 0.35 \text { a } \\
\begin{array}{l}0.66 \text { (muy } \\
\text { baja) }\end{array}\end{array}$ & 240 & 9.8 & 6762542 & 6.0 & 294943.51 & 15.1 \\
\hline
\end{tabular}


Tecnología y

Ciencias $₫$ Agua
2020, Instituto Mexicano de Tecnología del Agua

Open Access bajo la licencia CC BY-NC-SA 4.0

(https://creativecommons.org/licenses/by-nc-sa/4.0/)

\begin{tabular}{|l|c|c|c|c|c|c|}
\hline $\begin{array}{l}\text { De 0.67 a } \\
0.74 \text { (baja) }\end{array}$ & 488 & 19.9 & 14113314 & 12.6 & 335495.10 & 17.2 \\
\hline $\begin{array}{l}\text { De 0.75 a } \\
0.81 \text { (medio) }\end{array}$ & 847 & 34.5 & 29396290 & 26.2 & 824099.84 & 42.1 \\
\hline $\begin{array}{l}\text { De 0.82 a } \\
0.88 \text { (alto) }\end{array}$ & 723 & 29.4 & 62020698 & 55.2 & 469056.75 & 24.0 \\
\hline $\begin{array}{l}\text { De 0.89 a } \\
0.94 \text { (muy } \\
\text { alto) }\end{array}$ & 158 & 6.4 & 43694 & 0.0 & 32644.00 & 1.7 \\
\hline Total & 2456 & 100.0 & 112336538 & 100.0 & 1956239.19 & 100.0 \\
\hline
\end{tabular}

El estado con mayores problemas de seguridad hídrica por número de municipios es Oaxaca, donde hay 63 municipios con nivel muy bajo de seguridad, 119 con nivel bajo, 163 con nivel medio, 177 con nivel alto, y 48 con nivel muy alto.

Por extensión territorial de los municipios, el estado de Chihuahua encabeza la lista de mayor inseguridad hídrica, seguido de Durango y Guerrero. Por otro lado, los estados que resultan con mayor seguridad según la metodología propuesta son Coahuila (5 562.31 km²), Querétaro (3 $439.49 \mathrm{~km}^{2}$ ) y Veracruz (3 $055.46 \mathrm{~km}^{2}$ ).

En el nivel muy bajo de seguridad están 240 municipios (Tabla 8) del norte y sureste del país, con cerca de 6.373 millones de habitantes; en el nivel bajo, 488 municipios, con 13.301 millones de habitantes; en nivel alto de seguridad están 723 municipios, que comprenden el 55\% de la población del país, y en un nivel muy alto 158, con sólo 41179 habitantes. 


\section{Discusión}

La seguridad hídrica es transversal a todos los aspectos del desarrollo económico. Las personas asignan significado al concepto de seguridad hídrica dependiendo de la escala y el contexto particular en el que se aplica. La seguridad hídrica nunca podrá ser alcanzada totalmente, porque las condiciones físicas y económicas están en constante cambio, lo que requiere adaptación continua. No existe una solución única para aumentar la seguridad hídrica. Las soluciones deben adaptarse a las condiciones locales en cada país, cuenca, ciudad, proyecto o área de gestión. La seguridad hídrica y la gestión integrada de recursos hídricos (GIRH) son simbióticas. Por otra parte, la gestión adaptativa, inmersa en los procesos de la GIRH, ayuda a mejorar la seguridad hídrica del ámbito nacional al local. La seguridad hídrica debe ser vista como la meta de la GIRH. No se puede gestionar lo que no se puede medir y, por tanto, la medición de la seguridad hídrica es fundamental para aumentarla.

La tarea de alcanzar mayor seguridad hídrica supone responder no sólo a los riesgos que se observan en la actualidad, sino en forma muy importante dar respuestas adecuadas a los nuevos desafíos que presenta el sector. Los principales retos a vencer para alcanzar la seguridad hídrica se manifiestan en la escasez de agua, la contaminación de los cuerpos de agua, los efectos adversos de los fenómenos hidrometeorológicos 
extremos (inundaciones y sequías), los crecientes conflictos por el agua, y el deterioro ambiental de cuencas y acuíferos. Los factores principales que inducen o incrementan estos riesgos para la seguridad hídrica son los procesos demográficos; la creciente demanda de alimentos tanto por crecimiento demográfico como por cambios en la dieta; la demanda de agua para producción de energía; los efectos del cambio climático, y la deficiente gestión del agua.

La principal limitante para la construcción de un indicador apegado a la realidad en México - que además refleje claramente la situación del país - es la falta de información. Dicho rubro incluye la falta de medición, seguimiento y procesamiento de datos; información asimétrica a diferentes niveles de agregación (estatal y municipal), y el manejo discrecional de la información existente. Por otro lado, una complicación a la hora de realizar la integración de la información es determinar el grado de beneficio que aporta a la seguridad hídrica debido a la complejidad del problema.

Con respecto a los componentes que se analizaron para generar el indicador de seguridad hídrica, se puede concluir que van implícitos dentro de los indicadores considerados, y que inciden de forma transversal. Se recomienda incorporar en el futuro información relativa a los presupuestos autorizados y efectivamente erogados en los municipios relativos al control de inundaciones, sequías, tratamiento de agua, mejora de servicios de agua potable, etcétera; acciones que inciden directamente en el fortalecimiento de la seguridad hídrica del país. 


\section{Conclusiones}

El concepto de seguridad hídrica es variado según el contexto y la escala en que se maneje. No se espera alcanzar una totalidad de seguridad hídrica debido a los cambios constantes en todos los aspectos de la sociedad, pero es importante llevar a cabo acciones que ayuden a reducir el riesgo.

Los contrastes hidrológicos, meteorológicos y sociales a los que está expuesto el país hacen necesaria una sectorización espacial para analizar la seguridad hídrica; se ha considerado que una sectorización municipal permite la precisión adecuada para las variables que se han tomado en cuenta.

En este trabajo se propone una metodología para la generación de índices de seguridad bajo un enfoque probabilista en el ámbito municipal, compuesto por datos de los años 2010 a 2015, principalmente de Conagua para el peligro y del INEGI para la vulnerabilidad. El índice de peligro se compone de cuatro variables: inundaciones, sequías, falta de acceso a los servicios básicos de agua y abatimiento del nivel freático. En cuestión de vulnerabilidad, se toman en cuenta 13 indicadores; el riesgo se obtiene del producto de la vulnerabilidad y el riesgo. Por último, restando a uno el resultado anterior se genera el índice municipal. 
De los 2456 municipios de México, 240 (9.77\%) muestran un índice de seguridad hídrica muy riesgoso y 488 riesgoso (19.87\%), lo cual indica que casi $30 \%$ de los municipios de México tiene altas posibilidades de que se presenten fenómenos hidrometeorológicos extremos y tener pérdidas significativas y tener pérdidas significativas tanto de seres humanos como materiales.

Asimismo, por extensión territorial de los municipios, según el índice de seguridad hídrica, el estado de Chihuahua encabeza la lista de mayor inseguridad hídrica, seguido de Durango y Guerrero. Por otro lado, los estados que resultan con mayor seguridad según la metodología propuesta son Coahuila, Querétaro y Veracruz.

Existen parámetros que permiten calcular índices de seguridad hídrica en el nivel global (Gain, Giupponi, \& Wada, 2016); sin embargo, el índice propuesto en este trabajo toma en cuenta los indicadores que ya se miden en el país por instituciones gubernamentales, lo que facilita su aplicación en México. Sin embargo, no se descarta que una comparación entre estos índices podría aportar de una manera considerable al tema.

\section{Referencias}

Ait-Kadi, M., \& Linckaen, A. W. (2016). Aumentar la seguridad hídrica. un imperativo para el desarrollo. Recuperado de www.gwptoolbox.org.

AWDO, Asian Water Development Outlook. (2013). Asian water development outlook. Measuring water security in Asia and the Pacific. Manila, Philippines: Asian Development Bank. 
Cenapred-AR. (2014). Evaluación de la vulnerabilidad física y social. Guía básica para la elaboración del Atlas Nacional de Riesgos. Recuperado de http://www.cenapred.gob.mx/es/Publicaciones/archivos/57.pdf

Conagua-SMN, Comisión Nacional del Agua, Sistema Meteorológico Nacional. (2018). Servicio Meteorológico Nacional SMN. Obtenido del Monitor de Sequía de México (SMN). Recuperado de https://smn.conagua.gob.mx/es/climatologia/monitor-desequia/monitor-de-sequia-en-mexico

Conagua-Sina, Comisión Nacional del Agua, Sistema Nacional de Información del Agua. (2016). Declaratorias por fenómenos hidrometeorológicos (nacional). Recuperado de http://sina.conagua.gob.mx/sina/tema.php?tema=declaratoriasFe nomenos\&ver=mapa

Custodio, E. (2000). The complex concept of overexploited aquifer. Madrid, España: Fundación Marcelino Botín.

Gain, A. K., Giupponi, C., \& Wada, Y. (2016). Measuring global water security towards sustainable development. Environmental Research Letters, 11(12). DOI: 10.1088/1748-9326/11/12/124015

GWP, Global Water Partnership. (2012). La seguridad hídrica para el crecimiento y la sostenibilidad. Río + 20. Recuperado de www.gwptoolbox.org

GWP, Global Water Partnership. (2016). Aumentar la seguridad hídrica, un impertivo para el desarrollo. Global Water Partnership, Recuperado 
https://www.gwp.org/globalassets/global/toolbox/publications/per spective-papers/perspectives-paper_water-security_spanish.pdf

IMTA, Instituto Mexicano de Tecnología del Agua. (2017). Índices de seguridad hídrica (ISH), clave HC1711.1. Jiutepec, México: Secretaría de Medio Ambiente y Recursos Naturales, Instituto Mexicanano de Tecnología del Agua.

IMTA, Instituto Mexicano de Tecnología del Agua. (2018). Índices de Seguridad Hídrica (ISH). Jiutepec, México: Instituto Mexicano de Tecnología del Agua, INEGI, Instituto Nacional de Estadística y Geografía. (2019). Medición de la pobreza. Recuperado de https://www.coneval.org.mx/Medicion/Paginas/PobrezaInicio.aspx

Jenks, G. F. (1967). The data model concept in statistical mapping. International Yearbook of Cartography, 7, 186-190.

Liu, C., Tan, C. K., Fang, Y. S., \& Lok, T. S. (2012). The security risk assessement methodology. Procedia Engineering, 43, 600-609.

Martínez-Austria, P. F. (2013). Los retos de la seguridad hídrica. Tecnología y ciencias del agua, 4(5), 165-180.

Mason, N., \& Roger, (2012). Water security: From abstract concept to meaningful metrics. An initial overview of options. London, UK: Overseas Development Institute.

Norman, E., Bakker, K., Cook, C., Dunn, G., \& Allen, D. (2010). La sécurité hydrique: guide d'introduction, Élaboration d'un cadre stratégique en matiere de sécurité hydrique au Canada comme outil d'amélioration de la gouvernance de l'eau des bassans versants (2008-2012). 
http://watergovernance.sites.olt.ubc.ca/files/2010/05/WaterSecurity-Primer-FRENCH.pdf

OECD, Organization for Economic Cooperation and Development. (2008). Handbook on construction composite indicators-methology and user guide. Ispra, Italy: Organization for Economic Cooperation and Development.

Oliver-Smith, A., \& Hoffman, S. M. (2002). Introduction: Why antropologists should study disasters in catastrophe and culture: The anthropology of disaster. Recuperado de http://faculty.washington.edu/stevehar/O-S\&H.pdf

ONU, Organización de las Naciones Unidas-Agua. (2013). Water security and the global water agenda. A un-water analytical brief. Recuperado de https://collections. unu.edu/eserv/UNU:2651/Water-Security-andthe-Global-Water-Agenda.pdf

ONU, Organización de las Naciones Unidas. (2017). Objetivos del Desarrollo Sustentable. Recuperado de http://www.un.org/sustainabledevelopment/es/water-andsanitation/

Ortega-Gaucin, De la Cruz, B. J., \& Castellano, B. H. (2018). Peligro, vulnerabilidad y riesgo por sequía en el contexto del cambio climático en México. Recuperado de https://www.imta.gob.mx/biblioteca/libros_html/ccambioclimatico/agua-cambio-climatico.pdf 
Scalon, B., Longuevergne, L., \& Long, D. (2012). Ground referencing GRACE satellite estimates of groundwater storage changes in the California Central Valley, USA. Water Resources Research, 48(4), Recuperado de https://doi.org/10.1029/2011WR011312

Wahr, J., Zlotnick, V., \& Velicogna, I. (1998). Time variability of the Earth's gravity field: Hidrological and oceanic effects and their possible detection using GRACE. Journal of Geophysical Research: Solid Earth, 103(B12), Recuperado de https://doi.org/10.1029/98JB02844.

Wahr, J., Swenson, S., Zlotnicki, V., \& Velicogna, I. (2004). Time variable gravity from GRACE: First results. Geophysical Research Letters, 31 , L11501. Recuperado de https://doi.org/10.1029/2004GL019779 